\title{
Measuring Uncertainty about Long-Run Predictions
}

\author{
ULRICH K. MÜLLER \\ Princeton University, Department of Economics \\ and \\ MARK W. WATSON \\ Princeton University, Department of Economics
}

First version received September 2013; final version accepted December 2015 (Eds.)

\begin{abstract}
Long-run forecasts of economic variables play an important role in policy, planning, and portfolio decisions. We consider forecasts of the long-horizon average of a scalar variable, typically the growth rate of an economic variable. The main contribution is the construction of prediction sets with asymptotic coverage over a wide range of data generating processes, allowing for stochastically trending mean growth, slow mean reversion, and other types of long-run dependencies. We illustrate the method by computing prediction sets for 10- to 75-year average growth rates of U.S. real per capita GDP and consumption, productivity, price level, stock prices, and population.
\end{abstract}

Key words: Prediction interval, Low frequency, Spectral analysis, Least favourable distribution

JEL Codes: C22, C53, E17

\section{INTRODUCTION}

This article is concerned with quantifying the uncertainty in long-run predictions of economic variables. Long-run forecasts and the uncertainty surrounding them play an important role in policy, planning, and portfolio decisions. For example, in the U.S., an ongoing task of the Congressional Budget Office (CBO) is to forecast productivity and real GDP growth over a 75year horizon to help gauge the solvency of the Social Security Trustfund. Uncertainty surrounding these forecasts is then translated into the probability of trust fund insolvency 1 Inflation "Caps" and "Floors" are option-like derivatives with payoffs tied to the average value of price inflation over the next decade; their risk-neutral prices are determined by the probability that the long-run average of future values of inflation falls above or below a pre-specified threshold 2 And, there is a large literature in finance discussing optimal portfolio allocations for long-run investors and how these portfolios depend on uncertainty in long-horizon returns 3

1. See Congressional Budget Office 2005).

2. See Fleckenstein et al. 2013, Hilsher et al. 2014, and Kitsul and Wright 2013, who use market prices on various inflation-related derivatives to estimate market-based predictive distributions of inflation.

3. See, for example, Campbell and Viceira 1999, Pastor and Stambaugh 2012), and Siegel 2007 . 
Let $x_{t}$ denote a time series, such as the inflation rate, the growth rate of real GDP or the return on a portfolio of stocks. Sample data on $x_{t}$ are available for $t=1, \ldots, T$, say 1947-2014. Let $\bar{x}_{T+1: T+h}=h^{-1} \sum_{t=1}^{h} x_{T+t}$ denote the average value of the series between time periods $T+1$ through $T+h$, say the 32-year horizon 2014-46. We are interested in the date $T$ uncertainty about the value of $\bar{x}_{T+1: T+h}$, as characterized by prediction sets that contain $\bar{x}_{T+1: T+h}$ with a pre-specified probability (such as 90\%). This is a long-horizon problem, since the horizon $h$ is large relative to the number of available observations $T$ (in the example, $r=h / T \approx 0.5$ ).

We structure the problem so that the coverage probability can be calculated using asymptotic approximations based on a central limit theorem. In particular, we suppose that both $T$ and $h$ are large, and construct the prediction sets as a function of a relatively small number of weighted averages of the sample values of $x_{t}$. We apply a central limit theorem to the variable of interest $\left(\bar{x}_{T+1: T+h}\right)$ and the predictors, and study an asymptotic version of the prediction problem based on the multivariate normal distribution. Were all the parameters of this normal distribution known (or consistently estimable), the prediction problem would be a straightforward application of optimal prediction in the multivariate normal model.

The problem is complicated by unknown parameters that characterize the stochastic process $x_{t}$ and hence also the covariance matrix of the normal distribution in the large-sample problem. We assume that the first differences $\Delta x_{t}=x_{t}-x_{t-1}$ are covariance stationary. (Recall that $x_{t}$ is a series like the growth rate of real GDP, inflation, or asset returns, so this does not rule out stochastic trends in these growth rates.) Since we are interested in a long-run prediction $\left(\bar{x}_{T+1: T+h}\right.$, for $h$ large relative to $T$ ) the crucial characteristic of $x_{t}$ is its (pseudo-) spectrum near frequency zero. The relative paucity of sample information about these low-frequency properties precludes a non-parametric approach. We, therefore, proceed by constructing a flexible parametric model for the shape of the spectrum near frequency zero that nests the fractional, local-to-unity and local-level forms of long-run persistence. The uncertainty about the parameter $\theta$ of this model in turn becomes an important component of the uncertainty about $\bar{x}_{T+1: T+h}$.

We use both Bayes and frequentist methods to incorporate this uncertainty in our prediction sets. The Bayes procedure is straightforward: given a prior for the parameter $\theta$, and the Gaussianity of the limiting problem, the predictive density for $\bar{x}_{T+1: T+h}$ follows from Bayes rule, so that prediction sets are readily computed. While Bayes sets have many desirable properties, they have the potentially undesirable property of controlling coverage (i.e., the probability that the set includes the future value of $\bar{x}_{T+1: T+h}$ ) only on average for values of $\theta$ drawn from the prior. Thus in general, coverage will fall short of the nominal level for some values of $\theta$, and the specifics of this undercoverage will depend on the prior used. To address this limitation, we robustify the Bayes prediction sets by enlarging them so that they have frequentist properties: the resulting sets provide (possibly conservative) coverage for all values of $\theta$. Using ideas borrowed from Müller and Norets 2012), we do this in a way that minimizes the sets' average expected length.

In economics, arguably the most well-known predictive densities and corresponding prediction sets are the "Rivers of Blood" shown in the Bank of England's Inflation Report. These are judgmental prediction sets for inflation that are computed over a 4-year horizon by the members of the Bank's Monetary Policy Committee. In contrast, we are interested in prediction sets computed from probability models over long horizons, and the literature on this topic is relatively sparse. Most of the existing literature on long-horizon forecasting stresses the difficulty of constructing good long-term forecasts under uncertainty about the long-run properties of the process. Granger and Jeon 2007) provide a mostly verbal account. Elliott 2006) compares alternative approaches to point forecasts and compares their mean squared errors. Kemp 1999), Phillips 1998), and Stock 1996, 1997) show that standard formulas for forecast uncertainty break down in the long-horizon local-to-unity model, but they do not provide constructive alternatives. 
In the related problem of estimating long-run impulse responses, Pesavento and Rossi 2006) construct confidence sets that account for uncertainty about the local-to-unity parameter. Chapter 8.7 in Beran 1994) discusses forecasting of fractionally integrated series, and Doornik and Ooms 2004 use an ARFIMA model to generate long-run uncertainty bands for future inflation, but without accounting for parameter estimation uncertainty. Two strands of literature study longrun forecast uncertainty for time series that we analyse by constructing series-specific Bayesian models. Pastor and Stambaugh 2012) compute predictive variances of long-run forecasts of stock returns that account for parameter uncertainty. Lee 2011) and Raftery et al. 2012) study long-run forecasts of population and fertility rates.

The outline of this article is as follows. Section 2 formalizes the long-horizon prediction problem and discusses the low-frequency summaries of the sample data used in the analysis. This section also introduces two running examples: forecasting the average growth rate of real per capita GDP and the average level of consumer price index (CPI) inflation in the U.S. over the next 25 years. Section 3 discusses and develops the requisite statistical tools for constructing the long-horizon prediction sets. Two sets of tools are needed. The first is a central limit theorem and associated covariance matrix that yields a large-sample Gaussian version of the prediction problem. The second are methods for constructing Bayes and frequentist prediction sets for this limiting problem. The Bayes procedures are standard; the frequentist procedures are not, and are developed in Section 3.3 Section 4 takes up the important practical problems of parameterizing the covariance matrix in the limiting problem (which involves parameterizing the spectrum of $x_{t}$ near frequency 0), choosing a prior for the Bayes prediction sets and a related weighting function for the frequentist sets (to obtain a scalar criterion for comparing the efficiency of sets), and choosing the number of low-frequency averages of the sample data to use (which involves a classic trade-off between efficiency and robustness). Taken together, Sections 2 - 4 develop methods for constructing prediction sets with well-defined large-sample optimality properties; these methods are illustrated using the GDP and inflation running examples throughout these sections. Section 5 uses simulations and pseudo-out-of-sample experiments to evaluate the performance of these sets in small samples. One focus of this analysis is the effect of level and/or volatility "breaks" on the prediction sets. Following this extensive background, Section 6 applies these methods to construct prediction sets spanning up to 75 years for eight U.S. economic time series: the running examples of real GDP and CPI inflation, the rates of growth of per capita consumption expenditures, total factor and labour productivity, population, stock prices, and an alternative measure of price inflation. Section 7 concludes.

\section{THE PREDICTION PROBLEM}

Let $x_{t}$ be the economic variable of interest which is observed for $t=1, \ldots, T$. The objective is to construct a prediction set, denoted by $A$, of the average value of $x_{t}$ from periods $T+1$ to $T+h$,

$$
\bar{x}_{T+1: T+h}=h^{-1} \sum_{t=1}^{h} x_{T+t}
$$

with the property that $P\left(\bar{x}_{T+1: T+h} \in A\right)=1-\alpha$, where $\alpha$ is a pre-specified constant. The prediction set $A$ is constructed using the sample data for $x_{t}$, so that $A=A\left(\left\{x_{t}\right\}_{t=1}^{T}\right)$ 而 We restrict $A$ in two ways. First, we allow $A$ to depend on the sample data only through a small number low-frequency

4. Of course, when $x_{t}$ is the first difference of another variable $y_{t}$, so that $x_{t}=y_{t}-y_{t-1}$, then forecasts of $y_{T+h}$ can be constructed from forecasts of $\bar{x}_{T+1: T+h}$ using the identity $y_{T+h}=y_{T}+h \bar{x}_{T+1: T+h}$. Moreover, prediction sets for $\bar{x}_{T+1: T+h}$ 
weighted averages of the sample data, and secondly, we restrict $A$ to be scale and location equivariant. We discuss each of these restrictions in turn.

Cosine transformations of the sample data: Because $h$ is large, the prediction sets involve longrun uncertainty about $x_{t}$. It is, therefore, useful to transform the sample data into weighted averages that capture variability at different frequencies—we will be interested in the weighted averages corresponding to low frequencies. Thus, consider the weighted averages $\left(\bar{x}_{1: T}, \mathbf{X}_{T}\right)$, with $\bar{x}_{1: T}=$ $T^{-1} \sum_{t=1}^{T} x_{t}, \mathbf{X}_{T}=\left(X_{T}(1), \ldots, X_{T}(T-1)\right)^{\prime}$, and where $X_{T}(j)$ is the $j$-th cosine transformation

$$
X_{T}(j)=\int_{0}^{1} \Psi_{j}(s) x_{\lfloor s T\rfloor+1} d s=\iota_{j} T T^{-1} \sum_{t=1}^{T} \Psi_{j}\left(\frac{t-1 / 2}{T}\right) x_{t}
$$

with $\Psi_{j}(s)=\sqrt{2} \cos (j \pi s)$ and $\iota_{j T}=(2 T / j \pi) \sin (j \pi / 2 T) \rightarrow 1$. The cosine transforms have two properties that we will exploit. First, they isolate variation in the sample data corresponding to different frequencies: $\bar{x}_{1: T}$ captures zero-frequency variation and $X_{T}(j)$ captures variation at frequency $j \pi / T$. Secondly, because the $\Psi_{j}$ weights add to zero, $X_{T}(j)$ is invariant to location shifts of the sample, a property we use when we construct equivariant prediction sets.

The $T \times 1$ vector $\left(\bar{x}_{1: T}, \mathbf{X}_{T}\right)$ is a non-singular transformation of the sample data $\left\{x_{t}\right\}_{t=1}^{T}$, but we will construct prediction sets based on a truncated information set that includes only $\bar{x}_{1: T}$ and the first $q$ cosine transforms, $\mathbf{X}_{T, 1: q}=\left(X_{T}(1), X_{T}(2), \ldots, X_{T}(q)\right)^{\prime}$ and where $q$ is much smaller than $T-1$. Thus, the prediction sets we consider are of the form $A=A\left(\bar{x}_{1: T}, \mathbf{X}_{T, 1: q}\right)$, and so rely solely on variability in the data associated with frequencies lower than $q \pi / T$. We compress the sample information into the $q+1$ variables $\left(\bar{x}_{1: T}, \mathbf{X}_{T, 1: q}\right)$ for two reasons. The first is tractability: with a focus on this truncated information set, the analysis involves a small number of variables (the $(q+2)$ variables $\left.\left(\bar{x}_{T+1: T+h}, \bar{x}_{1: T}, \mathbf{X}_{T, 1: q}\right)\right)$, and because each of these variables is a weighted average of $\left\{x_{t}\right\}_{t=1}^{T+h}$, a central limit theorem derived in the next section allows us to study a limiting Gaussian version of the prediction problem that is much simpler than the original finite-sample problem. The second motivation for truncating the information set is robustness: we use the low-frequency information in the sample data $\left(\bar{x}_{1: T}\right.$ and the first $q$ elements of $\left.\mathbf{X}_{T}\right)$ to inform us about a low-frequency, long-run average of future data, but we do not use high-frequency sample information (the last $T-1-q$ elements of $\mathbf{X}_{T}$ ). While high-frequency information is informative about low-frequency characteristics for some stochastic processes (e.g. tightly parameterized ARMA processes), this is generally not the case, and high-frequency sample variation may lead to faulty low-frequency inference. Müller and Watson 2013, 2013) discuss this robustness issue in detail. In Section 4 below, we present numerical calculations that quantify the efficiencyrobustness trade-off embodied by the choice of $q$ in the long-run prediction problem.

Invariance: In our applications, it is natural to restrict attention to prediction sets that are invariant to location and scale, so for example, the results will not depend on whether the data are expressed as growth rates in percentage points at an annual rate or as percent per quarter. Thus, we restrict attention to prediction sets with the property that if $y \in A\left(\bar{x}_{1: T}, \mathbf{X}_{T, 1: q}\right)$ then $m+b y \in A\left(m+b \bar{x}_{1: T}, b \mathbf{X}_{T, 1: q}\right)$ for any constants $m$ and $b \neq 0$ (where the transformation of $\mathbf{X}_{T, 1: q}$ does not depend on $m$ because, as mentioned above, $\mathbf{X}_{T, 1: q}$ is location invariant). Invariance allows us to restrict attention to prediction sets that depend on functions of the sample data that are scale and location invariant; in particular, we can limit attention to constructing prediction

and $y_{T+h}$ are readily converted into prediction sets for monotonic transformation of these variables. For example, a prediction set for the average growth rate of real GDP $\left(\bar{x}_{T+1: T+h}\right)$ yields a prediction set for the log-level of real GDP $\left(y_{T+h}\right)$ or the level of real GDP $\left(\exp \left(y_{T+h}\right)\right)$. 
(a) Growth rate of real per-capita GDP

(i) Series and low-frequency component

(ii) Cosine transform
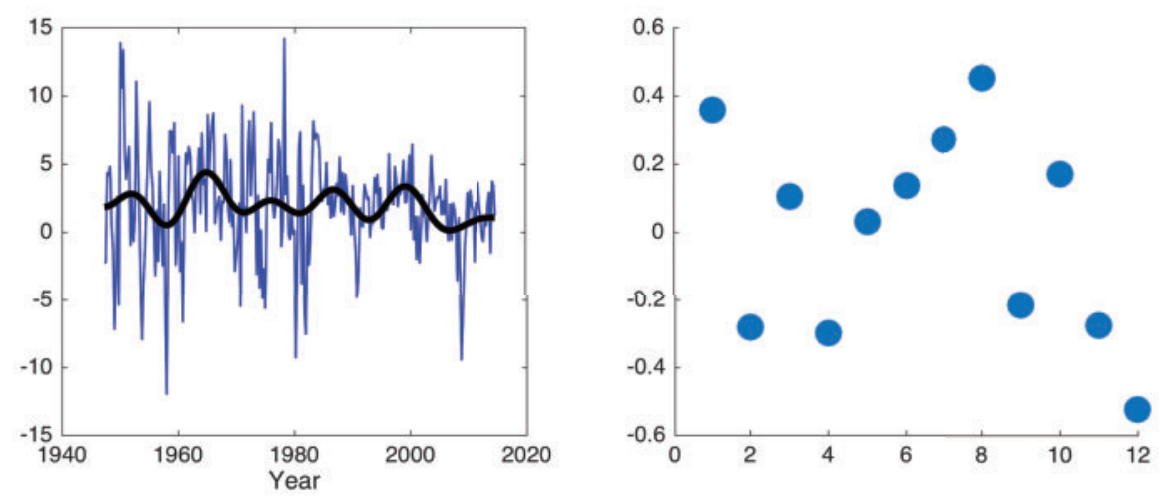

(b) Inflation (CPI)

(i) Series and low-frequency component

(ii) Cosine transform
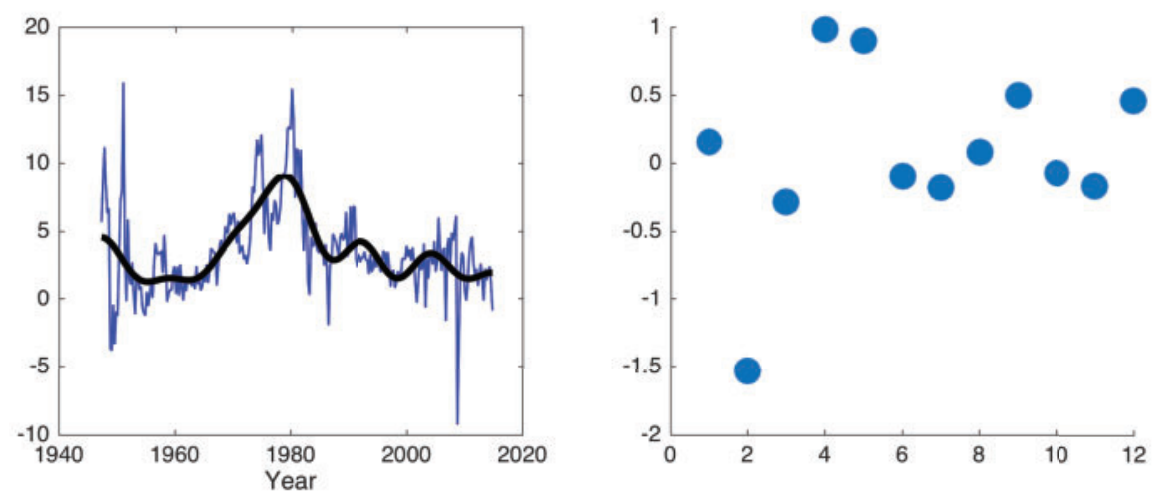

FIGURE 1

Low-frequency components and cosine transforms.

Notes: The low-frequency components in (i) are the projection of the series onto $\cos [(t-0.5) \pi j / T]$ for $j=0, \ldots, 12$. The cosine transforms shown in (ii) are the standardized values, $\boldsymbol{X}_{T, 1: q}^{s}$.

sets for $Y_{T}^{s}$ given $\mathbf{X}_{T, 1: q}^{s}$, where $Y_{T}^{s}=Y_{T} / \sqrt{\mathbf{X}_{T, 1: q}^{\prime} \mathbf{X}_{T, 1: q}}$ with

$$
Y_{T}=\bar{x}_{T+1: T+h}-\bar{x}_{1: T}
$$

and $\mathbf{X}_{T, 1: q}^{s}=\mathbf{X}_{T, 1: q} / \sqrt{\mathbf{X}_{T, 1: q}^{\prime} \mathbf{X}_{T, 1: q}}$

Running examples: Two of the economic time series studied in Section 6 are the growth rate of U.S. real per capita GDP and the rate of inflation in the U.S. based on the CPI. We use these series as running examples to illustrate concepts as they are introduced. Panels (i) in Figure 1 plot the

5. Setting $m=-\bar{x}_{1: T} \sqrt{\mathbf{X}_{T, 1: q}^{\prime} \mathbf{X}_{T, 1: q}}$ and $b=1 / \sqrt{\mathbf{X}_{T, 1: q}^{\prime} \mathbf{X}_{T, 1: q}}$ implies that for any invariant set $A, y \in$ $A\left(\bar{x}_{1: T}, \mathbf{X}_{T, 1: q}\right)$ if and only if $\left(y-\bar{x}_{1: T}\right) / \sqrt{\mathbf{X}_{T, 1: q}^{\prime} \mathbf{X}_{T, 1: q}} \in A\left(0, \mathbf{X}_{T, 1: q} / \sqrt{\mathbf{X}_{T, 1: q}^{\prime} \mathbf{X}_{T, 1: q}}\right)$, and thus also $\bar{x}_{T+1: T+h} \in$ $A\left(\bar{x}_{1: T}, \mathbf{X}_{T, 1: q}\right)$ if and only if $Y_{T}^{s} \in A\left(0, \mathbf{X}_{T, 1: q}^{s}\right)$. 
quarterly values of these time series from 1947 to 2014, along with the low-frequency components of the time series formed as the projection of the series onto $\cos [(t-1 / 2) \pi j / T]$ for $j=0, \ldots, 12$. (The value $q=12$ is used in the empirical analysis in Section 6 for reasons discussed below.) The coefficients in the projection are the cosine transformations, $\mathbf{X}_{T, 1: q}$, and their standardized values, $\mathbf{X}_{T, 1: q}^{s}$ are plotted in panels (ii). These low-frequency components of the data are the summaries of the sample data we use to construct long-horizon prediction sets. Looking at panels (i), inflation exhibits much more low-frequency variation than GDP growth rates over the sample period; this is manifested in panels (ii) by the relatively larger magnitude of inflation's first few cosine transformations, capturing pronounced low-frequency movements.

\section{STATISTICAL PRELIMINARIES}

The last section laid out the finite-sample prediction problem. In this section, we review and develop the statistical theory that will guide our approach to constructing prediction sets. We divide the section into three subsections. The first provides a central limit theorem that characterizes the large-sample behaviour of the weighted averages $\left(\mathbf{X}_{T, 1: q}, Y_{T}\right)$, and provides a characterization of the limiting covariance matrix based on the properties of the (pseudo-) spectrum of $x_{t}$ near frequency zero. The second subsection illustrates this framework in the fractional $I(d)$ model and reports prediction sets for known $d$ and Bayes prediction sets using a prior for $d$. The final subsection discusses the generic problem of robustifying Bayes prediction sets to obtain sets with frequentist coverage uniformly over the parameter space.

\subsection{Large-sample approximations}

To derive the asymptotic behaviour of $\left(\mathbf{X}_{T, 1: q}, Y_{T}\right)$, note that each element can be written as a weighted average of $x_{t}, t=1, \ldots, T+h$. Thus, let $g:[0,1+r] \mapsto \mathbb{R}$ denote a generic weighting function, where $r=\lim _{T \rightarrow \infty}(h / T)>0$, and consider

$$
\eta_{T}=T^{1-\kappa} \int_{0}^{1+r} g(s) x_{\lfloor s T\rfloor+1} d s
$$

for a suitably chosen constant $\kappa$. In our context, the elements of $\mathbf{X}_{T, 1: q}$ are cosine transformations of the in-sample values of $x_{t}$ (cf. equation (2) ), so that $g(s)=\sqrt{2} \cos (j \pi s)$ for $0 \leq s \leq 1$ and $g(s)=0$ for $s>1 ; Y_{T}$ defined in equation 3 is the difference between the out-of-sample and in-sample average values of $x_{t}$, so that $g(s)=-1$ for $0 \leq s \leq 1$ and $g(s)=r^{-1}$ for $1<s \leq 1+r$. These weights integrate to zero, so that the (unconditional) expectation of $x_{t}$ plays no role in the study of $\eta_{T}$.

In Appendix $\mathrm{A}$ we provide a central limit theorem for $\eta_{T}$ under a set of primitive conditions about the stochastic process describing $x_{t}$ and these weighting functions. We will not list the technical conditions in the text, but rather give a brief overview of the key conditions before stating the limiting result and discussing the form of the limiting covariance matrix. In particular, the analysis is carried out under the assumption that $\Delta x_{t}=\Delta x_{T, t}$ is a double array process with moving average representation $\Delta x_{T, t}=c_{T}(L) \varepsilon_{t}$, where $\varepsilon_{t}$ is a possibly conditionally heteroskedastic martingale difference sequence with more than two unconditional moments, which allows for some forms of short memory stochastic volatility 6 The moving average coefficients in $c_{T}(L)$ are square summable for each $T$, so that $\Delta x_{T, t}$ has a spectrum, denoted by $F_{T}(\lambda)$. The motivation

6. The restriction $E\left[\Delta x_{t}\right]=0$ rules out a deterministic trend in $x_{t}$. This restriction is plausible in our empirical analysis in which $x_{t}$ denotes growth rates of real variables like per capita GDP, inflation rates, and asset returns. 
for allowing $c_{T}(L)$ and $F_{T}$ to depend on $T$ is to capture many forms of persistence, as stemming from an autoregressive root local-to-unity, $\rho_{T}=1-c / T$, for instance.

The main regularity condition of the central limit theorem concerns the behaviour of the (pseudo-) spectrum of $x_{T, t}, R_{T}(\lambda)=F_{T}(\lambda) /\left|1-e^{-i \lambda}\right|^{2}$, for frequencies close to zero. (In general, $R_{T}$ is only a pseudo spectrum, since $\int_{-\pi}^{\pi} R_{T}(\lambda) d \lambda$ might not exist; for instance, it does not if $\Delta x_{T, t}$ is white noise, so that $x_{T, t}$ is a random walk.) In particular, we assume that there exists a function $S: \mathbb{R} \mapsto \mathbb{R}$ such that for all fixed $K>0$,

$$
\lim _{T \rightarrow \infty} \int_{0}^{K}\left|T^{1-2 \kappa} R_{T}\left(\frac{\omega}{T}\right)-S(\omega)\right| d \omega \rightarrow 0
$$

where $S$ is such that $\int_{0}^{\infty} \omega^{2} S(\omega) d \omega<\infty$. Intuitively, $S$ describes the limiting behaviour of $R_{T}$ close to frequency zero, and we correspondingly denote it as the "local-to-zero" spectrum.

Under these and additional technical assumptions, Theorem 1 in the Appendix A shows that $\eta_{T}$ has a limiting normal distribution 7 and as an implication

$$
T^{1-\kappa}\left[\begin{array}{c}
\mathbf{X}_{T, 1: q} \\
Y_{T}
\end{array}\right] \Rightarrow\left[\begin{array}{c}
\mathbf{X} \\
Y
\end{array}\right] \sim \mathcal{N}(0, \Sigma) \sim \mathcal{N}\left(0,\left(\begin{array}{cc}
\Sigma_{X X} & \Sigma_{X Y} \\
\Sigma_{Y X} & \Sigma_{Y Y}
\end{array}\right)\right)
$$

with $\mathbf{X}=\left(X_{1}, \ldots, X_{q}\right)^{\prime}$ (we omit the dependence of $\mathbf{X}$ on $q$ to ease notation). The asymptotic covariance matrix $\Sigma$ is a function of the local-to-zero spectrum $S$, as discussed further below.

The limiting density of the invariants $\mathbf{X}_{T, 1: q}^{s}=\mathbf{X}_{T, 1: q} / \sqrt{\mathbf{X}_{T, 1: q}^{\prime} \mathbf{X}_{T, 1: q}}$ and $Y_{T}^{s}=Y_{T} / \sqrt{\mathbf{X}_{T, 1: q}^{\prime} \mathbf{X}_{T, 1: q}}$ follows directly from $[6$ and the continuous mapping theorem,

$$
\left[\begin{array}{c}
\mathbf{X}_{T, 1: q}^{s} \\
Y_{T}^{s}
\end{array}\right] \Rightarrow\left[\begin{array}{c}
\mathbf{X}^{s} \\
Y^{s}
\end{array}\right]=\left[\begin{array}{l}
\mathbf{X} / \sqrt{\mathbf{X}^{\prime} \mathbf{X}} \\
Y / \sqrt{\mathbf{X}^{\prime} \mathbf{X}}
\end{array}\right]
$$

Note that as a consequence of the imposed scale invariance, the convergence in equation (7) holds irrespective of the scaling factor $\kappa$ in Equation (4), and the distribution of $\left(\mathbf{X}^{s}, Y^{s}\right)$ does not depend on the scale of $\Sigma$. Explicit expressions for the densities $f_{\mathbf{X}^{s}}$ and $f_{\left(\mathbf{X}^{s}, Y^{s}\right)}$ of $\mathbf{X}^{s}$ and $\left(\mathbf{X}^{s}, Y^{s}\right)$ as a functions of $\Sigma_{X X}$ and $\Sigma$ are provided in Appendix B

With $\Sigma$ known, it is straightforward to compute prediction sets of $Y^{s}$ given $\mathbf{X}^{s}=\mathbf{x}^{s}$ : a calculation shows that the distribution of $Y^{s}$ conditional on $\mathbf{X}^{s}=\mathbf{x}^{s}$ satisfies (see Appendix B

$$
\frac{Y^{s}-\Sigma_{Y X} \Sigma_{X X}^{-1} \mathbf{x}^{s}}{\sqrt{\Sigma_{Y Y}-\Sigma_{Y X} \Sigma_{X X}^{-1} \Sigma_{X Y}} \sqrt{\mathbf{x}^{s \prime} \Sigma_{X X}^{-1} \mathbf{x}^{s} / q}} \sim \text { Student- } t^{q}
$$

so that prediction sets for $Y^{s}$ of a given level $1-\alpha$ are readily computed using Student- $t$ quantiles. These sets in turn imply asymptotically justified prediction sets for $Y_{T}^{S}$ via equation (7), and thus also for $\bar{x}_{T+1: T+h}$ via the definition of $\left(\mathbf{X}_{T, 1: q}^{s}, Y_{T}^{s}\right)$ and equation (3).

In particular, when $x_{T, t}$ is $I(0)$ with long-run variance $\sigma^{2}$ (i.e. the local-to-zero spectrum is flat, $\left.S(\omega)=(2 \pi)^{-1} \sigma^{2}\right)$, it turns out that $\Sigma_{Y X}=0, \Sigma_{X X}=\sigma^{2} I_{q}, \Sigma_{Y Y}=\left(1+r^{-1}\right) \sigma^{2}$, and the

7. As in any central limit theorem, the conditions underlying Theorem 1 imply that no single shock has a substantial impact on the overall variability of $\eta_{T}$. This assumption might be violated by rare but catastrophic events stressed in the work of Riet 1988 and Barro 2006, for example. Note, however, that such events would need to substantially impact the average $\bar{x}_{T+1: T+h}$ over a long horizon to invalidate a normal approximation. 
$1-\alpha$ prediction set for $Y$ is given by the interval with endpoints $\pm t_{(1-\alpha / 2)}^{q} \sqrt{q\left(1+r^{-1}\right) \mathbf{X}^{\prime} \mathbf{X}}$, where $t_{(1-\alpha / 2)}^{q}$ is the $(1-\alpha / 2)$ quantile of the Student- $t$ distribution with $q$ degrees of freedom. The asymptotically justified prediction set for $\bar{x}_{T+1: T+h}$ is, therefore, $\bar{x}_{1: T} \pm t_{(1-a / 2)}^{q}(1+$ $\left.r^{-1}\right)^{1 / 2} T^{-1 / 2} s_{L R}$, where $s_{L R}^{2}=(T / q) \mathbf{X}_{T, 1: q}^{\prime} \mathbf{X}_{T, 1: q}$. Note that this interval becomes smaller for a larger horizon $r$ because a law of large numbers effect reduces the variability of the average of future values, with the residual uncertainty under $r \rightarrow \infty$ stemming from sampling uncertainty about the population mean $E\left[x_{t}\right]$.

More generally, the asymptotic covariance matrix $\Sigma$ can always be expressed as a function of the local-to-zero spectrum $S$ and the weighting functions $g_{j}$ that correspond to the $j$-th element of $\left(\mathbf{X}^{\prime}, Y\right)^{\prime}$. In particular, Corollary 1 of Appendix $\triangle$ implies that

$$
\Sigma_{j, k}=\int_{0}^{\infty} S(\omega) w_{j k}(\omega) d \omega
$$

where $w_{j k}(\omega)=2 \operatorname{Re}\left[\left(\int_{0}^{1+r} g_{j}(s) e^{-i \omega s} d s\right)\left(\int_{0}^{1+r} g_{k}(s) e^{i \omega s} d s\right)\right]$. The elements of the covariance matrix of $\left(\mathbf{X}^{\prime}, Y\right)^{\prime}$ are thus weighted averages of the local-to-zero spectrum $S$, with weights $w_{j k}(\omega)$ that are functions of the Fourier transforms of the weights $g_{j}(\cdot)$ used to construct $\mathbf{X}$ and $Y$.

The weights $w_{j k}(\omega)$ are plotted in the Supplementary Appendix; we highlight three features here. First, $w_{j k}(\omega)$ with $j \neq k$, integrates to zero, which implies that for a flat local-to-zero spectrum $S$ (corresponding to an $I(0)$ model), $\Sigma$ is diagonal, as already noted above. Secondly, the weight associated with the predictor $X_{j}$ is mostly concentrated in the interval $\pi j \pm 2 \pi$, so the variance of $X_{j}$ is determined by the value of $S$ in this frequency band. Thirdly, the weight associated with $Y$ has its mass concentrated near $\omega=0$; for example, when $r=1 / 2$, the variance of $Y$ is mostly determined by the shape of $S$ on the interval $\omega \in[0,4 \pi]$. The implication of these results is that the conditional variance of $Y$ given $\mathbf{X}$ depends on the local-to-zero spectrum, with the shape of $S$ for, say, $\omega<12 \pi$, essentially determining its value, even for large $q$. In terms of the original time series, frequencies of $|\omega|<12 \pi$ correspond to cycles of periodicity $T / 6$. For instance, with 60 years worth of data (of any sampling frequency), the shape of the spectrum for frequencies below 10 -year cycles essentially determines the uncertainty of the forecast of mean growth over the next 30 years.

\subsection{Prediction sets in the $I(d)$ model}

A leading example of this analysis is given by the fractional $I(d)$ model, which has a (pseudo-) spectrum proportional to $|\lambda|^{-2 d}$ for $\lambda$ close to zero; this yields the local-to-zero spectrum $S(\omega) \propto$ $|\omega|^{-2 d}$, and the central limit result from the last subsection is applicable for $-1 / 2<d<3 / 2$. The $I(d)$ model captures a wide range of long-run dependence patterns including the usual $I(0)$ and I(1) models, but also persistence patterns between and outside these two extremes. With negative values of $d$ it also allows for long-run anti-persistence (which may arise from overdifferencing), and with $d>1$ it allows for processes more persistent than an $I(1)$ process 8

Running example (continued): Panels (i) of Figure 2 show the appropriately centred and scaled Student- $t$ predictive densities from equation (8) for the average growth rate of U.S. real per capita GDP and the average value of CPI inflation over the next 25 years for various values of $d$ in the $I(d)$ model. For real GDP growth rates, predictive densities are shown for $d=-0.4,0.0,0.2$, and 0.5 , and for inflation the predictive densities are shown for $d=0.0,0.4,0.7$, and 1.0. For both 
(a) Growth rate of real per-capita GDP

(i) 25-year ahead $I(d)$-predictive densities

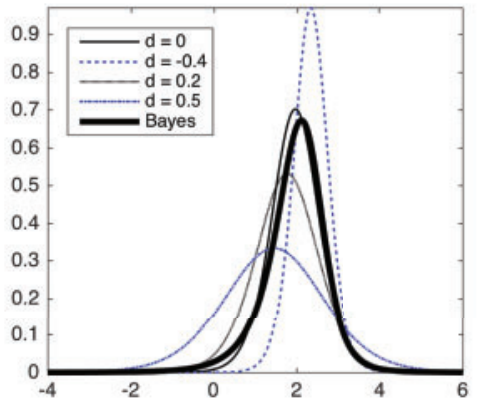

(ii) Low-frequency log-likelihood values for $d$

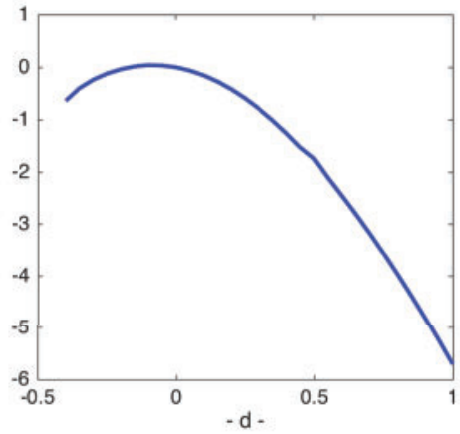

(b) Inflation (CPI)

(i) 25-year ahead $I(d)$-predictive densities

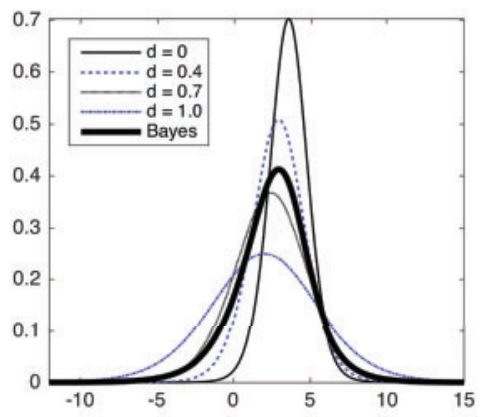

(ii) Low-frequency log-likelihood values for $d$

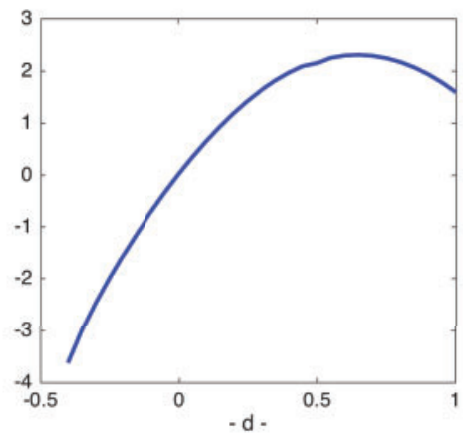

FIGURE 2

Predictive densities and low-frequency log-likelihood values for the $I(d)$ model.

Notes: Panels (i) show the known- $d$ prediction sets and Bayes prediction sets using the prior $d \sim \mathrm{U}[-0.4,1.0]$. The lowfrequency $I(d)$ likelihood is computed using $\boldsymbol{X}_{T, 1: q}^{s}$ and its asymptotic distribution given in the text; values are relative to the $I(0)$ model.

series, as $d$ increases, the variance of the predictive density increases because more persistence leads to larger variability in future average growth. The mode of the $I(0)$ predictive density is given by the in-sample mean (see the discussion following equation (8), and the mode shifts to the left for $d>0$ reflecting the persistent effect of the slow growth and low inflation experienced at the end of sample. In contrast, the mode of the $d=-0.4$ predictive density (shown for real GDP growth rates) is larger than the in-sample mean because faster than average growth is required to return the log-level of GDP to its pre-Great Recession trend growth path.

Evidently, both the length and location of 25-year-ahead prediction sets depend critically on the $d$. This raises the question: what is the value of $d$ for these series?

Panels (ii) summarize what the sample data say about the value of $d$. It plots the "lowfrequency" log-likelihood values for $d$ based on $\mathbf{X}_{T, 1: 12}^{s}$ and its large-sample density from equation (7), and with the log-likelihood of the $I(0)$ model normalized to zero. The numbers for real per capita GDP suggest only limited persistence for this series (values of $d>0.6$ yield a log-likelihood 3 points lower than the $I(0)$ model), but values of $d$ ranging from -0.4 (suggesting 
some reversion to a linear trend in the log-level of GDP, so that the growth rate is overdifferenced) to 0.2 (slight persistence in the GDP growth rates) all fit the data reasonably well. In contrast, the inflation data suggest much more persistence: the likelihood has a maximum at $d=0.65$ with corresponding log-likelihood value that is 2.3 points larger than the $I(0)$ model.

Taken together, the results in panels (i) and (ii) indicate that much of the 25 -year-ahead forecast uncertainty is associated with uncertainty about the degree of persistence in the stochastic process, which in the $I(d)$ model is governed by the value of the parameter $d$.

Bayes prediction sets: A natural way to incorporate this parameter uncertainty is to use a Bayes approach, where the limited-sample information is combined with a prior on $d$. This is straightforward: with $\Gamma$ the prior on $d$, the Bayes predictive density for $Y^{s}$ conditional on $\mathbf{X}^{s}=\mathbf{x}^{s}$ is given by

$$
f_{Y^{s} \mid \mathbf{X}^{s}}^{\Gamma}\left(y^{s} \mid \mathbf{x}^{s}\right)=\frac{\int f_{\left(\mathbf{X}^{s}, Y^{s}\right) \mid d}\left(\mathbf{x}^{s}, y^{s}\right) d \Gamma(d)}{\int f_{\mathbf{X}^{s} \mid d}\left(\mathbf{x}^{s}\right) d \Gamma(d)}
$$

with $f_{\left(\mathbf{X}^{s}, Y^{s}\right) \mid d}$ and $f_{\mathbf{X}^{s} \mid d}$ the densities of $\left(\mathbf{X}^{s}, Y^{s}\right)$ and $\mathbf{X}^{s}$ in equation 77 with the value of $\Sigma$ implied by a local-to-zero spectrum $S(\omega)$ proportional to $|\omega|^{-2 d}$.

Bayes prediction sets can be readily computed from the predictive density. For example, the "highest predictive density" (HPD) set for $Y^{s}$ is $A^{\mathrm{HPD}}\left(\mathbf{x}^{s}\right)=\left\{y^{s}: f_{Y^{s} \mid \mathbf{X}^{s}}^{\Gamma}\left(y^{s} \mid \mathbf{x}^{s}\right)>\operatorname{cv}\left(\mathbf{x}^{s}\right)\right\}$, where $\operatorname{cv}\left(\mathbf{x}^{s}\right)$ solves $\int_{A^{\mathrm{HPD}}\left(\mathbf{x}^{s}\right)} f_{Y^{s} \mid \mathbf{X}^{s}}^{\Gamma}\left(y^{s} \mid \mathbf{x}^{s}\right) d y^{s}=1-\alpha$. This HPD Bayes set is the smallest length set that satisfies the coverage constraint relative to $f_{Y^{s} \mid \mathbf{X}^{s}}^{\Gamma}$. Alternative Bayes prediction sets, such as equal-tailed sets, can be used instead. Thus, let $A^{\text {Bayes }}\left(\mathbf{x}^{s}\right)$ denote a generic Bayes prediction set for $Y^{s}$ as a function of $\mathbf{x}^{s}$. Because $Y^{s}=Y / \sqrt{\mathbf{x}^{\prime} \mathbf{x}}$ and $\mathbf{x}^{s}=\mathbf{x} / \sqrt{\mathbf{x}^{\prime} \mathbf{x}}$, equivariance implies the extension to generic $\mathbf{x}$ via $A^{\text {Bayes }}(\mathbf{x})=\left\{y: y / \sqrt{\mathbf{x}^{\prime} \mathbf{x}} \in A^{\text {Bayes }}\left(\mathbf{x} / \sqrt{\mathbf{x}^{\prime} \mathbf{x}}\right)\right\}$.

Running example (continued): Panels (i) of Figure 2 shows the resulting Bayes predictive densities for $\bar{x}_{T: T+h}$ with a uniform prior on $d \in[-0.4,1.0]$. This mixture of Student- $t$ densities is no longer necessarily symmetric, as the the underlying Student- $t$ densities do not have the same mode. So for instance, for the GDP series, one obtains a left-skewed Bayes predictive distribution since larger values of $d$ both increase uncertainty and shift the most likely future values to the left.

\subsection{Frequentist robustification of Bayes prediction sets}

As discussed in Section 3.1 the distributions of $(\mathbf{X}, Y)$ and $\left(\mathbf{X}^{s}, Y^{S}\right)$ depend on the covariance matrix $\Sigma$, which in turn depends on the low-frequency spectrum $S$ of $x_{t}$. In the next section, we discuss a parameterization of the spectrum that is more general than the $I(d)$ model, so in general, $\Sigma=\Sigma(\theta)$ where $\theta$ is a parameter vector. In this section, we discuss the general problem of constructing frequentist prediction sets that incorporate uncertainty about the value of $\theta$. We provide additional details in Appendix C

The (frequentist) coverage probability of a set $A, P_{\theta}(Y \in A(\mathbf{X}))$, generally depends on the value $\theta$. A Bayes prediction set has coverage probability of $1-\alpha$, on average relative to the prior $\Gamma$, that is $\int P_{\theta}\left(Y \in A^{\text {Bayes }}(\mathbf{X})\right) d \Gamma(\theta)=1-\alpha$, but in general, $P_{\theta}\left(Y \in A^{\text {Bayes }}(\mathbf{X})\right)<1-\alpha$ for some values of $\theta$. In this subsection, we "robustify" Bayes sets by enlarging them so they have frequentist coverage: $\inf _{\theta \in \Theta} P_{\theta}(Y \in A(\mathbf{X})) \geq 1-\alpha$. There is no unique way to achieve this. We focus on sets with smallest weighted expected length.

To be specific, let $A(\mathbf{X})$ denote an arbitrary prediction set, and $V_{\theta}(A)=E_{\theta}[\operatorname{vol}(A(\mathbf{X}))]$ denote its expected length (which depends on $\theta$ ). The goal is to choose $A$ to minimize $V_{\theta}(A)$ over the parameter space $\Theta$ for $\theta$. In many problems, including the one considered in this article, there is no set $A$ that simultaneously minimizes $V_{\theta}(A)$ for all $\theta \in \Theta$ while maintaining coverage, so there 
is an inherent trade-off of expected length over different values of $\theta$. Let $W$ denote a weighting function that makes this trade-off explicit. Consider the following problem:

$$
\min _{A} \int V_{\theta}(A) d W(\theta)
$$

subject to

$$
\begin{aligned}
\text { Equivariance: } & y \in A(\mathbf{x}) \text { implies } b y \in A(b \mathbf{x}) \text { for all } \mathbf{x}, y \text { and }|b| \neq 0 \\
\text { Frequentist coverage: } & \inf _{\theta \in \Theta} P_{\theta}(Y \in A(\mathbf{X})) \geq 1-\alpha, \text { and } \\
\text { Bayes superset: } & A^{\text {Bayes }}(\mathbf{x}) \subset A(\mathbf{x}) \text { for all } \mathbf{x} .
\end{aligned}
$$

Because the objective function depends on the weighting function $W$, so will the solution, and we discuss specific choices for $W$ in the following section. The constraint 11 imposes scale equivariance-recall that location invariance in the original problem is imposed by the choice of $Y$ and $\mathbf{X}$. The coverage constraint that defines a $(1-\alpha)$-frequentist prediction set is given by equation 12 .

The constraint 13 can be motivated in a variety of ways. One motivation is ad hoc and simply says that the goal is to robustify a Bayes set by enlarging it so it has frequentist coverage properties. Another focuses on properties of frequentist sets that do not impose constraint [13]. Notably, conditional on particular realizations of $\mathbf{X}$ these sets can have unreasonably small length; indeed they can be empty. In particular, even with $\theta$ known (i.e. $\Theta=\{\theta\}$ ), solving equation (10) subject to equations (11) and (12) does not in general yield the known- $\theta$ prediction set (8), but rather a prediction set whose coverage of $Y$ is equal to $1-\alpha$ only on average over repeated draws of $\mathbf{X}$, but not conditional on the observed $\mathbf{X}$. Müller and Norets 2012) show that imposing constraint (13) eliminates these arguably unattractive properties. We find the Müller and Norets arguments compelling and, therefore, enforce the constraint 130 for the frequentist sets used in the empirical analysis of Section 6 However, for comparison we also study solutions that do not impose constraint (13) in Section 4 and the Supplementary Appendix.

The solution to the program (10)-13) can be found in three steps: the first step transforms the problem to impose equivariance [11; the second uses a "least favourable distribution" for $\theta$ to simplify the coverage constraint 12]; and the third enforces constraint 13. We discuss these steps in turn.

Equivariance: If $A(\mathbf{X})$ is scale equivariant, then $Y \in A(\mathbf{X})$ if and only if $Y \in \sqrt{\mathbf{X}^{\prime} \mathbf{X}} A\left(\mathbf{X}^{s}\right)$. Thus, $\operatorname{vol}(A(\mathbf{X}))=\sqrt{\mathbf{X}^{\prime} \mathbf{X}} \operatorname{vol}\left(A\left(\mathbf{X}^{s}\right)\right)$ and $V_{\theta}(A)=E_{\theta}\left[g_{\theta}\left(\mathbf{X}^{s}\right) \operatorname{vol}\left(A\left(\mathbf{X}^{s}\right)\right)\right]$, where $g_{\theta}\left(\mathbf{X}^{s}\right)=$ $E_{\theta}\left[\sqrt{\mathbf{X}^{\prime} \mathbf{X}} \mid \mathbf{X}^{s}\right]$. Imposing this restriction, the objective function (10) becomes

$$
\min _{A} \int E_{\theta}\left[g_{\theta}\left(\mathbf{X}^{s}\right) \operatorname{vol}\left(A\left(\mathbf{X}^{s}\right)\right)\right] d W(\theta)
$$

and the coverage (12) and Bayes superset 13 constraints can be rewritten as

$$
\begin{gathered}
\inf _{\theta \in \Theta} P_{\theta}\left(Y^{s} \in A\left(\mathbf{X}^{s}\right)\right) \geq 1-\alpha \\
A^{\text {Bayes }}\left(\mathbf{x}^{s}\right) \subset A\left(\mathbf{x}^{s}\right) \text { for all } \mathbf{x}^{s} .
\end{gathered}
$$

Note that equations 14 16 only involve the value of $A$ evaluated at $\mathbf{x}^{s}$, which lives on a smaller subspace $\mathbf{x}^{s /} \mathbf{x}^{s}=1$ compared to $\mathbf{x} \in \mathbb{R}^{q}$, but on that subspace, $A$ is unrestricted. The 
solution to equation (14) subject to equations (15) and 116, $A^{*}\left(\mathbf{x}^{s}\right)$, then implies the solution $A^{*}(\mathbf{x})=\left\{y: y / \sqrt{\mathbf{x}^{\prime} \mathbf{x}} \in A^{*}\left(\mathbf{x} / \sqrt{\mathbf{x}^{\prime} \mathbf{x}}\right)\right\}$ to the original problem (10) subject to equations 111 13).

Frequentist Coverage: For the coverage constraint [15], suppose for a moment that $\theta$ is a random variable with distribution $\Lambda$, and consider solving equation (14) subject to the resulting single coverage constraint

$$
\int P_{\theta}\left(Y^{s} \in A\left(\mathbf{X}^{s}\right)\right) d \Lambda(\theta) \geq 1-\alpha .
$$

A calculations yields the solution

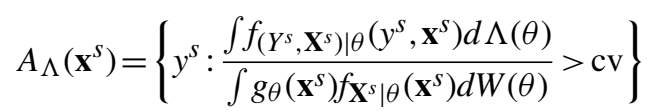

where cv is chosen to satisfy constraint 177 with equality. Of course, while $A_{\Lambda}$ satisfies the average coverage constraint [17, it does not necessarily satisfy the uniform coverage constraint 15] required for a frequentist prediction set. However, because any set satisfying constraint 15] also satisfies constraint [17, the value of the objective (14) evaluated at $A_{\Lambda}$ provides a lower bound for any set satisfying constraint 15 . Therefore, if a distribution $\Lambda^{\dagger}$ can be found under which $A_{\Lambda^{\dagger}}$ satisfies constraint [15], then $A_{\Lambda^{\dagger}}$ solves the minimization problem 14] subject to the uniform coverage constraint (15). Such a $\Lambda^{\dagger}$ is called the "least favourable distribution" for the problem. Elliott et al. 2015) develop numerical methods for approximating least favourable distributions in related problems, and we use a variant of those methods here. See the Supplementary Appendix for details.

Bayes superset: The final step-incorporating the constraint 16 —is straightforward: it simply amounts to replacing equation 18 with the set

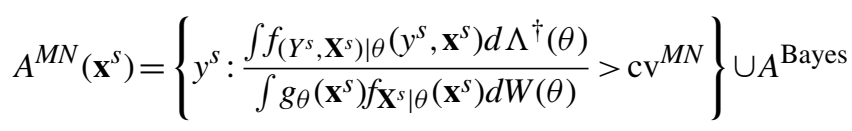

where $\left(\Lambda^{\dagger}, \mathrm{cv}{ }^{M N}\right)$ are now such that $\int P_{\theta}\left(Y^{s} \in A^{M N}\left(\mathbf{X}^{s}\right)\right) d \Lambda^{\dagger}(\theta)=1-\alpha$ and $\inf _{\theta \in \Theta} P_{\theta}\left(Y^{s} \in\right.$ $\left.A^{M N}\left(\mathbf{X}^{s}\right)\right) \geq 1-\alpha$ (cf. Müller and Norets, 2012).

\section{PARAMETERIZATIONS FOR LONG-HORIZON PREDICTION SETS}

Implementation of the prediction sets discussed in the last section requires four ingredients: (1) a parameterization of $S$, the local-to-zero spectrum, which yields the covariance matrix $\Sigma(\theta)$ via equation 19; (2) a Bayes prior $\Gamma(\theta)$, which yields the Bayes prediction set $A^{\text {Bayes; }}$ (3) a frequentist weighting function $W(\theta)$, which quantifies the trade-off of expected length for various values of $\theta$ in the objective function (10); and (4) a choice for $q$, the number of cosine weighted averages used for the prediction problem. These are discussed in the following three subsections.

\subsection{Parameterizing the low-frequency spectrum}

The $I(d)$ model introduced in Section 3.2 above is a flexible one-parameter model that captures a wide range of long-run persistence patterns. Because of its simplicity, flexibility, and use in other empirical analyses involving long-run behaviour of economic time series, we use the $I(d)$ parameterization for our equal-tailed Bayes prediction sets $A^{\text {Bayes }}$.

However, a concern is that the family of $I(d)$ local-to-zero spectra may not be sufficiently flexible to capture all forms of long-run dependencies in economic time series. This suggests 

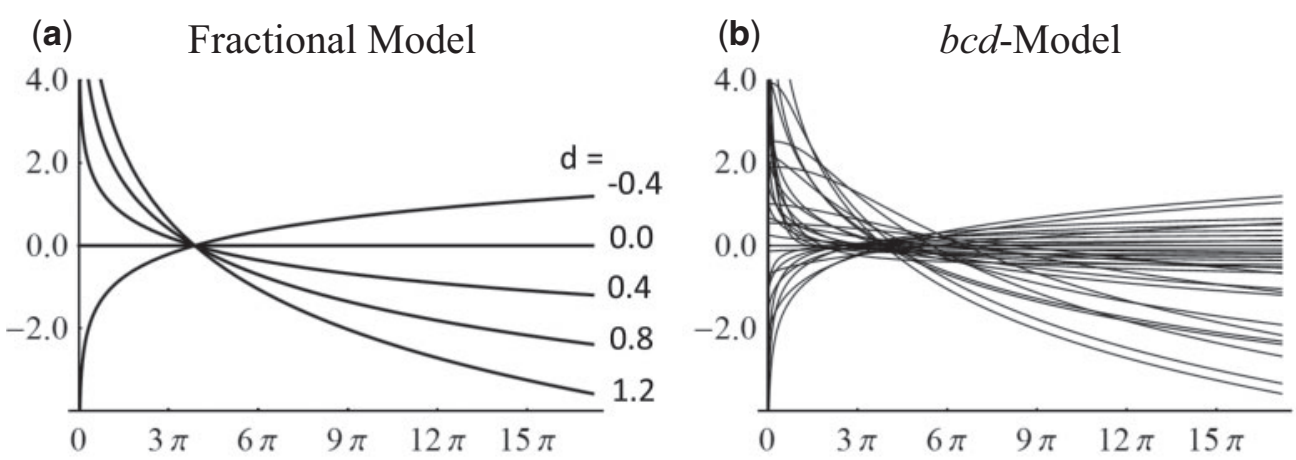

FIGURE 3

Logarithm of the local-to-zero spectrum for selected models.

Notes: The scale of the local-to-zero spectra $S$ are normalized such that $\ln (S(\pi))+\ln (S(15 \pi))=0$.

the need for a richer class of local-to-zero spectra, and we construct such a class by considering two other models that have proven useful for modelling low-frequency characteristics in other contexts. The first is the local-level model that expresses $x_{t}$ as the sum of an $I(0)$ process and an $I(1)$ process, say $x_{t}=e_{1 t}+(b T)^{-1} \sum_{s=1}^{t} e_{2 s}$, where $\left\{e_{1 t}\right\}$ and $\left\{e_{2 t}\right\}$ are mutually uncorrelated $I(0)$ processes with the same long-run variance. The $I(1)$ component has relative magnitude $1 / b$ and is usefully thought of as a stochastically varying "local mean" of the growth rate $x_{t}$, as arising from some forms of stochastic breaks. In this model, $S(\omega) \propto b^{2}+\omega^{-2}$. The second model is the local-to-unity AR(1) model, widely used to model highly persistent processes. In this model $x_{t}=(1-c / T) x_{t-1}+e_{t}$, where $e_{t}$ is an $I(0)$ process, and a straightforward calculation shows that $S(\omega) \propto 1 /\left(\omega^{2}+c^{2}\right)$. (Note that $(b, c) \rightarrow(\infty, \infty)$ and $(b, c) \rightarrow(0,0)$ recover the $I(0)$ and $I(1)$ model, respectively.) The $I(d)$, local-level and local-to-unity models are nested in the parameterization

$$
S(\omega) \propto\left(\frac{1}{\omega^{2}+c^{2}}\right)^{d}+b^{2}
$$

where $b=c=0$ for the $I(d)$ model, $d=1$, and $c=0$ for the local-level model, and $d=1$, and $b=0$ for the local-to-unity model 9

Figure 3 plots the logarithm of the local-to-zero spectrum of the $I(d)$ model in panel (a), and of this " $b c d$-model" in panel (b). The $b c d$-parameterization allows us to capture a wide range of monotone shapes for the low-frequency (pseudo-) spectrum of $x_{t}$, including, but not limited to, the three benchmark models discussed above. In the analysis below we let $\theta=(b, c, d)$, so that $\Sigma(\theta)$ is given by equation 9 with the local-to-zero spectrum $S$ as in equation 20 .

\subsection{Bayes and frequentist weighting functions}

In the empirical analysis in Section 6 we assume that $S$ is characterized by the $b c d$-model, with $-0.4 \leq d \leq 1.0$ and $b, c \geq 010$ As mentioned above, we construct Bayes sets using a prior that puts

9. This is recognized as the local-to-zero spectrum of the process $x_{t}=e_{1 t}+\left(b T^{d}\right)^{-1} z_{t}$, where $\left(1-\rho_{T} L\right)^{d} z_{t}=e_{2 t}$ with $\rho_{T}=1-c / T$ and $\left\{e_{1 t}\right\}$ and $\left\{e_{2 t}\right\}$ are mutually uncorrelated $I(0)$ processes with the same long-run variance. It is also recognized as the spectrum of the Whittle-Matérn process from spatial statistics (e.g. Lindgren, 2013). Autocovariances for this process are derived in the Supplementary Appendix

10. For the variables we study (growth rates of real variables, inflation rates, and asset returns), values of $d>1.0$ are unnecessary, but these values may be appropriate in other applications, and we note that the results in Section 3.1 hold for the $b c d$-model with $-0.5<d<1.5$, and $b, c \geq 0$. 
TABLE 1

Coverage for nominal $67 \%$ and $90 \%$ prediction sets, $r=0.5, q=12$

\begin{tabular}{|c|c|c|c|c|c|c|}
\hline & \multicolumn{3}{|c|}{$67 \%$ Prediction Sets } & \multicolumn{3}{|c|}{$90 \%$ Prediction Sets } \\
\hline & $A^{\text {Bayes }}$ & $A_{d}^{M N}$ & $A_{(b, c, d)}^{M N}$ & $A^{\text {Bayes }}$ & $A_{d}^{M N}$ & $A_{(b, c, d)}^{M N}$ \\
\hline$b=0, c=0, d \sim \mathrm{U}[-0.4,1.0]$ & 0.67 & 0.71 & 0.73 & 0.90 & 0.93 & 0.94 \\
\hline $\begin{array}{l}\text { Coverage minimized over: } \\
b=0, c=0,-0.4 \leqslant d \leqslant 1.0 \\
b \geqslant 0, c \geqslant 0,-0.4 \leqslant d \leqslant 1.0\end{array}$ & $\begin{array}{l}0.55 \\
0.54\end{array}$ & $\begin{array}{l}0.67 \\
0.57\end{array}$ & $\begin{array}{l}0.68 \\
0.67\end{array}$ & $\begin{array}{l}0.81 \\
0.81\end{array}$ & $\begin{array}{l}0.90 \\
0.84\end{array}$ & $\begin{array}{l}0.90 \\
0.90\end{array}$ \\
\hline
\end{tabular}

Notes: $A^{\text {Bayes }}$ is the equal-tailed Bayes prediction set using the prior $b=0, c=0, d \sim \mathrm{U}[-0.4,1.0] ; A_{d}^{M N}$ robustifies the Bayes set so it has frequentist coverage for $b=0, c=0, d \in[-0.4,1.0] ; A_{(b, c, d)}^{M N}$ robustifies the Bayes set so it has frequentist coverage for $b \geqslant 0, c \geqslant 0, d \in[-0.4,1.0]$

all weight on the $I(d)$ model (so that $b=c=0$ ); we use a prior with uniform weight on values of $d \in[-0.4,1.0]$. The $A^{M N}$ sets robustify these Bayes sets so they have frequentist coverage for all values of $b, c \geq 0$ and $d \in[-0.4,1.0]$. The analysis is usefully thought of in terms of the various spectral shapes plotted in Figure 3 and the Bayes prior is seen as putting equal weight on the various shapes in panel (a). Because $S$ may take on shapes other than those represented by the $I(d)$ models in panel (a), the $A^{M N}$ sets robustify the Bayes analysis to ensure frequentist coverage over all shapes shown in panel (b).

Construction of the $A^{M N}$ sets requires specification of the weighting function $W$ in equation (10). As noted in Section 3.3 the function $W$ determines the trade-off between expected length for various of $\theta$, which is necessary because there is no single prediction set that minimizes expected length for all $\theta$. Our choice of $W$ is guided by the observation that, even with $\theta$ known, the minimized values of $V_{\theta}(A)$ vary greatly over the values of $\theta$. For example, in the $I(d)$ model with known $d$, prediction sets are much wider when $d=1$ (so that $x_{t} \sim I(1)$ ) than when $d=0$ $\left(x_{t} \sim I(0)\right)$. To account for these differences, we scale $V_{\theta}(A)$ so that it is expressed in units of the expected length of the predictions set for known $\theta$. Denote this scaled version of $V_{\theta}(A)$ by $R_{\theta}(A)=V_{\theta}(A) / V_{\theta}^{\text {known }}$, where $V_{\theta}^{\text {known }}$ is the expected length of the prediction set for known value of $\theta$ implied by equation (8). In terms of $R_{\theta}(A)$ we use a weighting function that coincides with the Bayes prior: uniform on $d \in[-0.4,1.0]$ and with $b=c=0$ (so in terms of $V_{\theta}(A)$, the weighting function $W$ is proportional to $\left.1 / V_{\theta}^{\text {known }}\right) 11$

Table 1 shows coverage rates for $67 \%$ and $90 \%$ prediction sets for $h=r T$, with $r=0.5$ using $q=12$ cosine transforms. (This is the value of $q$ we will use in the empirical analysis, and is discussed more fully in the next subsection.) Table 1 answers two questions. First, what is the frequentist coverage of the Bayes prediction sets across the range of processes represented by the spectra in panels (a) and (b) of Figure 3? And secondly, does the $I(d)$ model provide sufficient flexibility so that the additional parameters $b$ and $c$ are unnecessary in practice? The table, therefore, displays coverage rates for three prediction sets: the Bayes set, $A^{\text {Bayes; }}$ the set robustified to have correct frequentist coverage over $d$ but with $b=c=0$, denoted $A_{d}^{M N}$; and the set robustified to have correct frequentist coverage over $(b, c, d), A_{(b, c, d)}^{M N}$. Coverage rates are shown for three configurations of $(b, c, d)$. In the first, values of $(b, c, d)$ are drawn from the prior, so $A^{\text {Bayes }}$ has correct coverage; in the second, the coverage probability is minimized over $-0.4 \leq d \leq 1.0$ with $b=c=0$, so $A_{d}^{M N}$ has correct coverage; and in the third, the coverage

11. We investigate how this weighting function performs relative to other possible weighting in the Supplementary Appendix where we also compute the cost (in terms of expected length) of the Bayes superset constraint 16. 
probability is also minimized over $b, c \geq 0$, so $A_{(b, c, d)}^{M N}$ has the correct coverage. The table indicates that $A^{\text {Bayes }}$ exhibits substantial undercoverage for some values of $d$ and $(b, c, d)$. It also indicates substantial undercoverage of $A_{d}^{M N}$ for some values of $(b, c, d)$. Evidently, controlling coverage over $d$ does not provide adequate coverage for long-run persistence patterns associated with nonzero values of $b$ and $c$. Thus, because some economic variables are arguably well described by stochastic processes with non-zero value of $b$ and $c$, it seems prudent to construct the $A_{(b, c, d)}^{M N}$ sets 12

We draw four conclusions from Table 1 and the analysis of $W$ detailed in the Supplementary Appendix. First, Bayes sets constructed using a uniform prior on $d$ exhibit substantial undercoverage for some values of $d$. Secondly, robustifying these sets to achieve frequentist coverage over $d$ is inadequate for some processes with non-zero values of $b$ and $c$. Thirdly, for many values of $(b, c, d)$ our benchmark choices of $\Gamma$ and $W$ produce sets with expected length close to the smallest achievable length under the coverage constraint. And finally, for most values of $(b, c, d)$ there is little cost in terms of expected length for constructing frequentist sets that are supersets of Bayes sets (and, therefore, share some their desirable properties).

\subsection{Choice of $q$}

As discussed in Section 2 the choice of $q$ may usefully be thought of as a trade-off between efficiency and robustness. In principle, the central limit theorem for $\left(\mathbf{X}_{T 1: q}^{\prime}, Y_{T}\right)^{\prime}$ discussed in Section 3.1 holds for any fixed $q$, at least asymptotically. And the larger $q$, the smaller the (average) uncertainty about $Y_{T}$. This suggests that one should pick $q$ large to increase efficiency of the procedure.

At the same time, one might worry that approximations provided by the central limit theorem for $\left(\mathbf{X}_{T 1: q}^{\prime}, Y_{T}\right)^{\prime}$ become poor for large $q$. The concern is not only that the high-dimensional multivariate Gaussianity might fail to be an accurate approximation; more importantly, any parametric assumption about the shape of the local-to-zero spectrum becomes stronger for larger $q$. In particular, for a given sample size $T$, the assumption that the spectrum of $x_{t}$ over the frequencies $[-q \pi / T, q \pi / T]$ is well approximated by the spectrum of the $b c d$-model becomes less plausible the larger $q$. Roughly speaking, we fit a parametric model to the $q$ observations $\mathbf{X}_{T, 1: q}$, so a concern about non-trivial approximation errors arises for large $q$, irrespective of the sample size $T$.

We are thus faced with a classic efficiency and robustness trade-off. Recall from the discussion in Section 3.1 that the object of interest - the variability of long-run forecasts, as embodied by the conditional variance of $Y$ given $\mathbf{X}$-is a low frequency quantity that is essentially governed by properties of $x_{t}$ over frequencies $[-12 \pi / T, 12 \pi / T]$. Since the predictors $X_{T}(j)$ provide information for frequency $j \pi T$, this suggests that the marginal benefit of increasing $q$ beyond $q=12$ is modest, at least with the spectrum known.

With the spectrum unknown, $\mathbf{X}$ with larger $q$ provides additional information about its scale and its shape. The scale effect is most easily understood in the $I(0)$ model. As discussed above, the $I(0)$ prediction set is $\bar{x}_{1: T} \pm t_{(1-a / 2)}^{q}\left(1+r^{-1}\right)^{1 / 2} T^{-1 / 2} s_{L R}$, where $s_{L R}^{2}=(T / q) \mathbf{X}_{T, 1: q}^{\prime} \mathbf{X}_{T, 1: q}$. The average asymptotic length of this forecast is thus $2 T^{-1 / 2} t_{(1-a / 2)}^{q}\left(1+r^{-1}\right)^{1 / 2} E \sqrt{\mathbf{X}^{\prime} \mathbf{X} / q}$ with

12. The approximate least favourable distributions (ALFDs) that underlie the $A_{(b, c, d)}^{M N}$ sets are plotted in the Supplementary Appendix The ALFD is non-degenerate and has most of its mass on spectra that are relatively flat for larger $\omega$, but with a pronounced pole at zero (these spectra arise, for instance, in the local-level model with moderate $b)$. Intuitively, in the local-level model, the strong mean reversion of the $I(0)$ component masks the pronounced long-run uncertainty, making it relatively hardest to control coverage. 
TABLE 2

25-year-ahead prediction sets

\begin{tabular}{|c|c|c|c|c|c|c|}
\hline \multirow[t]{2}{*}{ Series } & \multicolumn{3}{|c|}{$67 \%$} & \multicolumn{3}{|c|}{$90 \%$} \\
\hline & $A^{\text {Bayes }}$ & $A^{M N}$ & $A^{I(0)}$ & $A^{\text {Bayes }}$ & $A^{M N}$ & $A^{I(0)}$ \\
\hline GDP/Pop & $(1.3,2.6)$ & $(1.2,2.6)$ & $(1.4,2.5)$ & $(0.7,3.0)$ & $(-0.3,3.0)$ & $(0.9,3.0)$ \\
\hline Inflation & $(0.3,4.8)$ & $(0.3,4.8)$ & $(2.3,4.8)$ & $(-1.8,6.5)$ & $(-2.6,6.8)$ & $(1.4,5.7)$ \\
\hline
\end{tabular}

Notes: The table shows the prediction sets for the average value of the growth rate of real per capita GDP and inflation from 2014-2039 using the benchmark values of the $\Gamma, W$, and $q$.

$\mathbf{X} \sim \mathcal{N}\left(0, \sigma^{2} I_{q}\right)$, which decreases in $q$, since $t_{(1-a / 2)}^{q} E \sqrt{\mathbf{X}^{\prime} \mathbf{X} / q}$ is a decreasing function of $q 13$ But the benefit of increasing $q$ is modest: for a $90 \%$ interval, the average length for $q \in\{24,48, \infty\}$ is only $\{3.0 \%, 4.4 \%, 5.8 \%\}$ shorter than for $q=12$, for instance.

When the shape of the spectrum is unknown but parametrized, as in the $b c d$-model, increasing $q$ beyond 12 provides additional information about the shape of the spectrum over the crucial frequencies $[-12 \pi / T, 12 \pi / T]$. Table B.2 in the Supplementary Appendix quantifies the combined scale and shape effects by reporting the value of the objective $\int V_{\theta}(A) d W(\theta)$ in the program (10) for $q \in\{6,12,24,48\}$. In this $\theta$ unknown case, there is an $8 \%$ decrease in average length as $q$ increases from $q=12$ to $q=24$ and a further reduction of $5 \%$ for $q=48$.

In our view, these potential gains are still relatively moderate and do not outweigh concerns about spectral misspecification that arise with a large choice of $q$. We, therefore, suggest constructing the prediction sets by default with $q=12$, but also report results for different values of $q$ in Section 6 .

Running example (continued): Table 2 shows the $67 \%$ and $90 \% A^{\text {Bayes }}$ and $A^{M N} 25$-year-ahead predictions sets for real GDP growth and inflation using the benchmark values of the Bayes prior $(\Gamma)$, weighting function $(W)$, and $q=12$. The $67 \% A^{\text {Bayes }}$ and $A^{M N}$ sets coincide, while the $90 \%$ $A^{M N}$ sets are somewhat wider than the $A^{\text {Bayes }}$ sets. For comparison, the table also shows the prediction sets computed from the $I(0)$ model. These are similar to the $A^{\text {Bayes }}$ and $A^{M N}$ sets for GDP (although the 67\% I(0) set is shifted to the left for reasons discussed above), but are much different for inflation (where the $I(0)$ are shifted the right and are much narrower), and where both results are as expected given the predictive densities and log-likelihood values displayed in Figure 2 Section 6 discusses these empirical results in more detail.

\section{FINITE SAMPLE EXPERIMENTS}

In the last two sections, we developed a large-sample framework for constructing Bayes and frequentist long-run prediction sets that is tailored to models of long-run persistence typically used for economic time series. This large sample analysis is sufficiently general to allow for in-sample and out-of-sample stochastic breaks in the series, as long as these breaks occur with sufficient frequency that sample averages satisfy the central limit theorem discussed in Section 3 And the large-sample analysis also accommodates short-memory stochastic shifts in volatility. But does this large-sample analysis provide reliable prediction sets for the sample sizes and stochastic processes typically encountered in applied economics? This section addresses this question using two sets of finite sample experiments. The first set of experiments are Monte Carlo simulations in which we generate data with level and volatility breaks designed to mimic the kinds of breaks seen

13. This is analogous to the wider confidence intervals that arise from the use of inconsistent HAC estimators as developed by Kiefer et al. 2000 and Kiefer and Vogelsang 2005), for example; see Müller 2014) for a review. 
in some macroeconomic time series. The second set of experiments uses rolling samples of daily interest rates and stock returns to construct pseudo-out-of-sample prediction sets and uses actual values of these interest rates and returns to evaluate these sets. We discuss these experiments in the following two subsections.

\subsection{Monte Carlo simulations with breaks in level and volatility}

Post-sample breaks of arbitrary size can undermine any attempt at prediction, so the methods proposed here are not immune to arbitrarily defined breaks. That said, a more relevant concern is how well the methods fare in the face of breaks that plausibly have occurred in the kinds of series to which the methods are to be applied. We address that question in this subsection. Statistical characterizations of uncertainty require a probability framework, so we consider breaks that occur probabilistically. And, because of the macroeconomic applications we carry out in Section 6 the models for these breaks are motivated by the behaviour of important macroeconomic time economic series in the post-WWII U.S.

We consider five models. The first two involve breaks in the level of $x_{t}$

$$
x_{t}=\mu_{t}+u_{t}
$$

where $\mu_{t}$ denotes the "level" of $x_{t}$ and $u_{t}$ is a zero-mean stochastic process that is independent of $\mu_{t}$. We suppose that $\mu_{t}$ shifts discretely by an amount $\pm \delta$ at irregular time periods determined by the indicator $s_{t}$, so that

$$
\mu_{t}=\mu_{t-1}+s_{t} \delta_{t}
$$

where $s_{t}$ is an i.i.d. Bernoulli process with $P\left(s_{t}=1\right)=p$, and $\delta_{t}= \pm \delta$ with equal probability independent of $s_{t}$. Because $\mu_{t}$ follows a martingale, an $I(1)$ process, its sample averages are characterized by the Gaussian limits in Section 3 (as an $I(1)$ model for fixed $p, \delta>0$ and a special case of the local-level model in section 4 .1 for fixed $p>0$ and $\delta=O\left(T^{-1}\right)$ ). That said, when $p$ is small, shifts in $\mu_{t}$ occur infrequently and the finite sample behaviour of sample averages may be quite different from their large-sample Gaussian limit.

The second two models involve breaks in volatility. In these models, $x_{t}$ has components that can be represented as $\sigma_{t} e_{t}$, where $e_{t}$ is an $I(0)$ process and $\sigma_{t}$ is a volatility process that evolves as $\ln \left(\sigma_{t}\right)=\mu_{t}$, where $\mu_{t}$ follows equation 22). While the central limit used in Section 3 allows for certain forms of heteroscedasticity, it does not allow volatility to evolve as an $I(1)$ process. Thus, the volatility models in this section involve stochastic processes that are strictly more general than the processes analysed above, even in large samples.

The final model involves breaks in both the level and volatility of $x_{t}$. Specifically, following Pesaran et al. 2006) (also see Chib, 1998), $x_{t}$ follows a different AR(1) process (with intercept and possibly a unit root) within each of a sequence of regimes. Regimes end stochastically according the Bernoulli process $s_{t}$ above, although with a regime-specific value of $p$, and new regimes begin with new parameter values for the AR process and $p$ drawn from a fixed probability distribution.

We choose model parameters to match specific characteristics of post-WWII U.S. quarterly macroeconomic data. Thus, we chose $T$ to correspond to 65 years, and as above we consider forecast horizons of $h=0.5 T$ with $q=12$ and the prior $(\Gamma)$ and weighting function $(W)$ described in Section 4 For models 1-4, we choose two values for the break frequency: $p_{\text {large }}=1 / 40$ (so a break occurs, on average, once every 40 quarters) and $p_{\text {small }}=1 / 260$ (so a break occurs, on average, once during the sample period). The other parameter values depend on the experiment and are motivated by the behaviour of particular U.S. macroeconomic time series. 
Model 1 is motivated by the growth rate of average labour productivity, which visually appears to be an $I(0)$ process but around a time varying level (see the Supplementary Appendix Figure C.4). Labour productivity growth averaged $2.2 \%$ per year in the post-WWII period, but experienced decade-long swings that were roughly 1 percentage point higher (early 1960s and late 1990s) or lower (1970s and early 1980s) than the average. The first model, therefore, takes the form 21] with $u_{t} \sim \operatorname{iid} \mathcal{N}\left(0, \sigma_{u}^{2}\right)$, where $\sigma_{u}$ is chosen to match the long-run standard deviation of average labour productivity, and the magnitude of the breaks in $\mu_{t}$ was chosen to yield a sensible value for the interquartile range (IQR) of $\mu_{T}-\mu_{0}$. Specifically, for each value of $p$ we chose two values for $\delta$, where the first, $\delta_{\text {small }}$, yielded an IQR of $0.5 \%$ and the second, $\delta_{\text {large }}$, yielded an IQR of $1.5 \%$.

Model 2 is similar to Model 1, but is motivated by the behaviour of nominal interest rates, which follow a pattern consistent with equation (21) but with $u_{t}$ a highly serially correlated process. Thus for this experiment, $u_{t}$ was generated by an AR(1) process with coefficient 0.98 , Gaussian innovations with variance chosen to match 10-year U.S. Treasury Bonds, and $\delta_{\text {small }}$ and $\delta_{\text {large }}$ chosen so that the IQR for $\mu_{T}-\mu_{0}$ was $2.0 \%$ and $4.0 \%$, respectively.

Model 3 is designed to capture features in the data like the "Great Moderation": a lowfrequency reduction in the volatility in real U.S. macroeconomic variables. For example, the standard deviation of growth rates of measures of real aggregate activity (GDP, employment, etc.) fell rather abruptly by roughly $30 \%$ in the early $1980 \mathrm{~s}$ (e.g. Stock and Watson, 2002). Thus, in this model the data were generated as $x_{t}=\sigma_{t} e_{t}$, with $e_{t} \sim \operatorname{iid} \mathcal{N}(0,1)$ and $\ln \left(\sigma_{t}\right)=\mu_{t}$ generated as described above with $\delta_{\text {small }}$ and $\delta_{\text {large }}$ chosen so that the IQR for $\ln \left(\sigma_{T} / \sigma_{0}\right)$ was 0.25 and 0.75 , respectively.

Model 4 is designed to capture the changes in variability and persistence evident in the U.S. inflation process. Stock and Watson 2007), Cogley and Sargent 2014), and others argue that these features can be captured by a local-level model with stochastic volatility. Thus, in this model we generate data as $x_{t}=e_{1 t}+\sum_{s=1}^{t} \sigma_{s} e_{2 s}$, where $e_{1 t}$ and $e_{2 t}$ are mutually independent i.i.d. standard normal random variables, $\ln \left(\sigma_{t}\right)=\mu_{t}$ follows the process described above, and the parameters are chosen to mimic estimates of the time-varying $\operatorname{IMA}(1,1)$ representation of the model found in U.S. data (e.g. Watson, 2014). Specifically, $\sigma_{0}$ is chosen so that the MA coefficient is 0.5 in the initial period, and $\delta_{\text {small }}$ and $\delta_{\text {large }}$ were chosen so that the IQR of the full-sample change in the MA coefficient was 0.5 and 0.8 .

Model 5 uses parameter values from Pesaran et al. 2006) of their analysis of nominal U.S. Treasury Bill rates from 1947 to 2002 and, therefore, captures the changing volatility and persistence in post-WWII interest rates 14

Results for the various experiments are shown in Table 3 where panel (a) shows results for the $A^{\text {Bayes }}$ sets and panel (b) shows results for the $A^{M N}$ sets. The first row of each panel shows results for the model with $p=0$ (so that breaks are absent); the other rows show results for $p_{\text {small }}, p_{\text {large }}$, $\delta_{\text {small }}$ and $\delta_{\text {large }}$. When $p=0$, Models 1 and 3 are i.i.d. processes for which both $A^{\text {Bayes }}$ and $A^{M N}$ have coverage rates that exceed their nominal level. This overcoverage occurs because $A^{\text {Bayes }}$ provides correct average coverage for $I(d)$ processes that includes both small and large values of $d$, and coverage for small $d$ is less than the average coverage. Similar reasoning explains the overcoverage for $A^{M N}$, which is designed to achieve uniform coverage over $(b, c, d)$. And with $p=0$, Model 2 is well approximated by the local-to-unity model with $c=260(1-0.98)=5.2$ and Model 4 is well approximated by an $I(1)$ process; $A^{M N}$ satisfies the coverage constraint in both models, while $A^{\text {Bayes }}$ severely undercovers in model 4 , achieving the same undercoverage shown previously in Table 1 for the $I(d)$ model. Moving to the results with $p>0, A^{\text {Bayes }}$ has coverage 
TABLE 3

Coverage probability for simulated data, $\mathrm{T}=65$ years, $\mathrm{r}=0.5$, and $\mathrm{q}=12$

\begin{tabular}{|c|c|c|c|c|c|c|c|c|c|c|c|}
\hline \multirow[b]{2}{*}{ Nom. level } & & \multicolumn{2}{|c|}{$\begin{array}{l}\text { Model 1: } \\
\text { Break in } \\
\text { level, } I(0) \\
\text { model }\end{array}$} & \multicolumn{2}{|c|}{$\begin{array}{c}\text { Model 2: } \\
\text { Break in } \\
\text { level, AR(1) } \\
\text { model }\end{array}$} & \multicolumn{2}{|c|}{$\begin{array}{l}\text { Model 3: } \\
\text { Break in } \\
\text { volatility, } \\
I(0) \text { model }\end{array}$} & \multicolumn{2}{|c|}{$\begin{array}{c}\text { Model 4: } \\
\text { Break in volatility, } \\
I(0)+I(1) \\
\text { model }\end{array}$} & \multicolumn{2}{|c|}{$\begin{array}{l}\text { Model 5: } \\
\text { PPT Break } \\
\text { model }\end{array}$} \\
\hline & & $67 \%$ & $90 \%$ & $67 \%$ & $90 \%$ & $67 \%$ & $90 \%$ & $67 \%$ & $90 \%$ & $67 \%$ & $90 \%$ \\
\hline & \multicolumn{11}{|c|}{ (a) $A^{\text {Bayes }}$} \\
\hline & \multirow{3}{*}{$\begin{array}{c}\delta_{\text {small }} \\
\delta_{\text {large }}\end{array}$} & 0.72 & 0.94 & 0.65 & 0.89 & 0.72 & 0.94 & 0.55 & 0.81 & \multirow[t]{2}{*}{0.65} & \multirow[t]{3}{*}{0.88} \\
\hline \multirow{2}{*}{$p_{\text {large }}$} & & 0.68 & 0.92 & 0.64 & 0.89 & 0.71 & 0.94 & 0.57 & 0.77 & & \\
\hline & & 0.58 & 0.85 & 0.63 & 0.88 & 0.71 & 0.92 & 0.8 & 0.73 & \multirow{3}{*}{0.62} & \\
\hline \multirow[t]{3}{*}{$p_{\text {small }}$} & $\delta_{\text {small }}$ & 0.69 & 0.93 & 0.64 & 0.89 & 0.71 & 0.94 & 0.57 & 0.79 & & \multirow{2}{*}{0.80} \\
\hline & $\delta_{\text {large }}$ & 0.62 & 0.88 & 0.63 & 0.88 & 0.71 & 0.93 & 0.57 & 0.75 & & \\
\hline & \multicolumn{9}{|c|}{ (b) $A^{M N}$} & \multirow{4}{*}{0.72} & \multirow{4}{*}{0.93} \\
\hline$p=0$ & & 0.74 & 0.95 & 0.70 & 0.94 & 0.74 & 0.95 & 0.67 & 0.90 & & \\
\hline \multirow{2}{*}{$p_{\text {large }}$} & $\delta_{\text {small }}$ & 0.71 & 0.94 & 0.71 & 0.94 & 0.74 & 0.95 & 0.67 & 0.85 & & \\
\hline & $\delta_{\text {large }}$ & 0.67 & 0.90 & 0.70 & 0.93 & 0.73 & 0.94 & 0.65 & 0.79 & & \\
\hline \multirow[t]{2}{*}{$p_{\text {small }}$} & $\delta_{\text {small }}$ & 0.72 & 0.94 & 0.71 & 0.93 & 0.74 & 0.95 & 0.68 & 0.87 & \multirow[t]{2}{*}{0.68} & \multirow{2}{*}{0.85} \\
\hline & $\delta_{\text {large }}$ & 0.69 & 0.91 & 0.71 & 0.93 & 0.74 & 0.94 & 0.66 & 0.82 & & \\
\hline
\end{tabular}

Notes: The table shows coverage probability of $A^{\text {Bayes }}$ and $A^{M N}$ sets for four models subject to breaks in level (Models 1 and 2) or volatility (Models 3 and 4), or breaks and volatility (Model 5). In Models 1-4 breaks occur in each time period with probability $p$ and are of size $\delta_{\text {small }}$ or $\delta_{\text {large }}$. The models are described in the text. Models $1-4$ use 65 years of quarterly data. Model 5 uses 65 years of monthly data.

rates notably less than its nominal level in Models 4 and 5; coverage rates for nominal $67 \% A^{M N}$ are approximately correct for all models, but there is some undercoverage in Models 4 and 5 .

In summary, we conclude that the build-in safeguards against non-stationarities in our approach seem to be mostly adequate for series that are comparable to post-WWII U.S. macroeconomic series.

\subsection{Pseudo-out-of-sample forecasts}

The last section examined the performance of long-run prediction sets using simulated data, but how well do the sets perform for actual data? Ideally, pseudo-out-of-sample experiments could be used to answer this question using economic time series from a wide array of stochastic processes. However, this is difficult in our setting-where we are interested in long-horizon forecasts for macroeconomic series in developed economies like the U.S.- because the available macroeconomic data provide little pseudo-out-of-sample information.

But the salient definition of a long-run forecast is that the horizon is long relative to the sample data. And in contrast to macroeconomic data, there are long time series on high-frequency financial variables. One empirical test of the methods developed here is thus to see whether forecasts constructed from, say, daily observations from 1 year of financial data, have reasonable empirical coverage for forecasts of the average value over the following half year. We carry out two pseudo-out-of sample experiments.

For the first experiment, we use value-weighted S\&P daily returns, $r_{t}$, from CRSP from 1926 to 2014, for a total of 23,535 returns. The pseudo-out-of-sample exercise uses a rolling sample of $T=260$ observations to construct prediction sets for the average value of $r_{t}$ and $r_{t}^{2}$ over the next $h=0.5 T=130$ periods, where the choice of $T$ matches the sample size used in the last section and in much of the empirical analysis in Section 6 Rolling through the sample in this way allows us to compute 23,145 (or 178 non-overlapping) pseudo-out-of sample prediction sets. The second 
TABLE 4

Coverage rates for prediction sets in pseudo-out-of-sample experiments rolling sample, $T=260$ days, $q=12$ and $r=0.5$

\begin{tabular}{|c|c|c|c|c|c|c|}
\hline \multirow[t]{2}{*}{ Prediction set } & \multicolumn{2}{|c|}{ Returns } & \multicolumn{2}{|c|}{ Squared returns } & \multicolumn{2}{|c|}{$\begin{array}{l}\text { 3-month Treasury } \\
\text { Bill interest rate }\end{array}$} \\
\hline & $67 \%$ & $90 \%$ & $67 \%$ & $90 \%$ & $67 \%$ & $90 \%$ \\
\hline$A^{\text {Bayes }}$ & 0.71 & 0.92 & 0.64 & 0.84 & 0.53 & 0.73 \\
\hline$A^{M N}$ & 0.73 & 0.93 & 0.69 & 0.88 & 0.65 & 0.85 \\
\hline$A^{I(0)}$ & 0.67 & 0.88 & 0.42 & 0.69 & 0.11 & 0.24 \\
\hline
\end{tabular}

Notes: The table shows empirical coverage rates for $A^{\text {Bayes }}$ and $A^{M N}$ prediction sets in 23,145 (=178 non-overlapping) pseudo-out-of-sample periods for the average value of SP500 returns and squared returns, and 15,007 (=115 nonoverlapping) periods for the average value of 3-month Treasury Bill interest rate.

experiment is similar, but uses daily observations on nominal interest rates for 3-month U.S. Treasury Bills from 1954 to 2014.

Results for $67 \%$ and $90 \%$ prediction sets are summarized in Table 4 For the return series, both $A^{\text {Bayes }}$ and $A^{M N}$ have sample coverage rates slightly larger than their nominal values; this result is not unexpected given the results in the preceding sections. Squared returns are significantly more persistent than the level of returns, and are often given as an example of an economic time series that exhibits $I(d)$ low-frequency behaviour (see, for instance, Ding et al., 1993). Table4)indicates that the pseudo-out-of-sample coverage for $A^{\text {Bayes }}$ is slightly lower than its nominal level, while the coverage of $A^{M N}$ remains near its nominal level; again, these results are not unexpected given the simulation results of the last subsection. Daily values of nominal interest rates are highly persistent and exhibit shifting volatility; coverage rates for $A^{\text {Bayes }}$ are substantially below their nominal levels, while coverage rates for $A^{M N}$ are much closer to the nominal level; these results are broadly in line with those from Model 5 of the last section. In contrast, for squared returns and the interest rate series, forecast intervals computed from the $I(0)$ model have coverage far below nominal level, underlying the necessity to flexibly adjust to various forms of persistence.

\subsection{A final pseudo-out-of-sample forecast}

The results from the Monte Carlo simulations lead us to conclude that predictions sets based on asymptotic approximations developed in Sections 3 and parameterizations in Section 4 perform reasonably well in the face of the kinds of breaks that have occurred in the post-WWII U.S. macroeconomy. This conclusion is buttressed by the results from the pseudo-out-of-sample forecasts for daily asset returns and interest rates. Of course, this does not imply that these prediction sets will produce sensible ex post results in all circumstances, and we end this section with one example.

Data on per capita GDP suggests that the U.S. economy was dramatically more volatile in the pre-WWII period than after. For example, the standard deviation of annual per capita GDP growth rates fell from $7.8 \%$ over $1901-46$ to just $2.4 \%$ over $1947-2014$. Estimates of long-run standard deviations show a similar reduction $(8.5 \%$ falling to $2.6 \%)$. While the source of the decline is a matter of debate (see Balke and Gordon, 1989; Romen, 1989; and Watson, 1994 for discussion), imagine using the data from 1901 to 1946 to construct a prediction set for average growth over the following 46 years, from 1947 to 1992. Using the formula below equation 88,

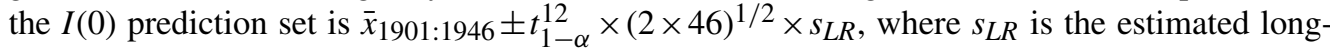
run standard deviation constructed from the pre-war data with $q=12$. Using $\bar{x}_{1901: 1946}=1.86 \%$ and $s_{L R}=8.54 \%$, the $67 \%$ prediction set is wide: $(0.1 \%, 3.7 \%)$. Indeed, given the low volatility experienced since 1947, the prediction set is implausibly wide; had it been constructed using the 
post-1946 value of $s_{L R}=2.6 \%$ it would have been much narrower, $(1.3 \%, 2.4 \%$ ). (The realized value of average GDP growth over $1947-1992$ was $\bar{x}_{1947: 1992}=2.1 \%$.)

What do we make of the 1946 prediction set? Here are two observations. First, there was considerable uncertainty about the future of U.S. growth following WWII, with many forecasters predicting a return to the growth patterns experienced during the 1930s and others predicting rapid growth (see Walton and Rockoff, 2013). The 1946 prediction set was arguably more plausible in 1946 than it is today. Secondly, while the Monte Carlo simulations suggested relatively small coverage distortions associated with low-frequency volatility shifts, these shifts (1) were not as large as the 2.5-fold decrease in volatility in post-WWII GDP and (2) were two-sided (volatility increases and decreases), while the single realization for GDP was necessarily one sided. A lesson from this example is that in some circumstances it may be important to explicitly incorporate large and potentially predictable breaks in volatility, and the required modifications are outlined in the article's final section.

\section{PREDICTION SETS FOR U.S. MACROECONOMIC TIME SERIES}

In this section, we present prediction sets for eight U.S. economic time series for forecast horizons ranging from 10 to 75 years using sample data through 2014. These series include the growth rate of per capita values of real GDP and CPI inflation used as the running examples, and also the growth rates of real per capita consumption expenditures, population, productivity (both total factor and labour productivity), real stock returns, and prices as measured by the PCE deflator. We construct prediction sets using post-WWII quarterly samples, and for several series, samples that extend into the early 20th century. We also examine prediction sets for inflation in Japan as a contrast to results for U.S. inflation. Sources and details of construction of the data are presented in the supplementary Data Appendix. Supplementary appendix Figures C.1-C.14 provide a variety of summary statistics for each series including a plot of the series, its low-frequency components, normalized cosine transformations, low-frequency $I(d)$ log-likelihood values, and $67 \%$ and $90 \%$ Bayes, $\mathrm{MN}$ and $I(0)$ prediction sets for all horizons between 10 and 75 years. Table 5 reports a summary of the prediction sets for prediction sets for 10-, 25-, 50-, and 75-year horizons.

We now discuss the results for specific series in more detail.

Real per capita GDP: The Bayes prediction sets for per capita GDP narrow as the forecast horizon increases, consistent with the reduction in variance of the sample mean for an $I(0)$ process. The frequentist sets coincide with the Bayes sets for (relatively) short horizons but include smaller values of average GDP growth rates at longer horizons. Apparently, to guarantee high coverage uniformly in the $b c d$-model at long horizons, the frequentist sets allow for the possibility of more persistence in the GDP process, so that the slow-growth rates of the past decade are predicted to potentially persist into the future. A comparison of the prediction sets constructed using the post-WWII data and the long annual (1901-2014) series shows that the pre-WWII data tend to widen the predictions sets, presumably because of the higher (long-run) variance in the pre-WWII data discussed above.

At the 75 -year horizon the $80 \%$ Bayes prediction interval is $1.3-2.5$, which roughly coincides with the $80 \%$ interval reported by the Congressional Budget Office 2005) for 75-year forecasts beginning in 2004. The coincidence of the Bayes/CBO sets arises despite important differences in the way they are computed. The CBO interval is based on simulations computed from its long-run model with inputs such as TFP growth simulated from estimated $I(0)$ models. The CBO interval differs from the Bayes interval in two important respects. First, because the simulations are carried out using fixed values of the model parameters, the CBO method ignores the parameter uncertainty in $\bar{x}_{1: T}$ (as an estimate of $\mu$ ) and $s_{L R}^{2}$ (as a an estimate of the long-run variance). Ignoring this uncertainty leads the $\mathrm{CBO}$ interval to underestimate uncertainty in the predictions. Secondly, 
TABLE 5

Prediction sets

\begin{tabular}{|c|c|c|c|c|}
\hline \multicolumn{5}{|c|}{ (a) $67 \%$ coverage } \\
\hline \multirow[t]{2}{*}{ Series } & \multicolumn{4}{|c|}{ Forecast horizon (in years) } \\
\hline & 10 & 25 & 50 & 75 \\
\hline \multicolumn{5}{|c|}{ Quarterly post-WWII series } \\
\hline GDP/Pop & (1.1 3.0) & $\begin{array}{l}(1.32 .6) \\
{[1.2,2.6]}\end{array}$ & $\begin{array}{l}(1.5,2.4) \\
{[0.7,2.4]}\end{array}$ & $\begin{array}{l}(1.52 .4) \\
{[0.5,2.4]}\end{array}$ \\
\hline Cons/Pop & $(1.23 .0)$ & $(1.42 .7)$ & $\begin{array}{l}(1.5,2.6) \\
{[1.2 .2 .6]}\end{array}$ & $\begin{array}{l}(1.6,2.5) \\
{[1.0 .25]}\end{array}$ \\
\hline TF prod & 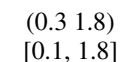 & $\begin{array}{c}(0.51 .8) \\
{[-0.2,1.9]}\end{array}$ & 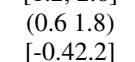 & $\begin{array}{c}(0.61 .8) \\
{[-0.6,2.4}\end{array}$ \\
\hline Labour prod & $\begin{array}{l}(0.82 .6) \\
{[0.8,2.7]}\end{array}$ & $(1.02 .6)$ & $\begin{array}{l}(1.22 .5) \\
{[0.8,2.7]}\end{array}$ & $\begin{array}{l}(1.32 .5) \\
{[0.6,2.8]}\end{array}$ \\
\hline Population & $\begin{array}{l}(0.6,1.0) \\
{[0.5,1.0]}\end{array}$ & $\begin{array}{l}(0.5,1.1) \\
{[0.4,1.2]}\end{array}$ & $\begin{array}{l}(0.4,1.2) \\
{[0.2,1.4]}\end{array}$ & $\begin{array}{l}(0.4,1.3) \\
{[0.0,1.5]}\end{array}$ \\
\hline Inflation (PCE) & $(0.23 .7)$ & $(0.0,4.1)$ & $(-0.24 .5)$ & $(-0.4,4.7)$ \\
\hline Inflation (CPI) & $(0.44 .5)$ & $(0.34 .8)$ & $(0.25 .1)$ & $\begin{array}{c}(0.1,5.3) \\
{[-0.1,5.3]}\end{array}$ \\
\hline Infl. (CPI,Japan) & $\begin{array}{l}(-1.4,4.4) \\
{[-1.7,4.4]}\end{array}$ & $\begin{array}{l}(-1.7,4.9) \\
{[-2.4,4.9]}\end{array}$ & $\begin{array}{l}(-2.1,5.4) \\
{[-3.8,5.4]}\end{array}$ & $\begin{array}{l}(-2.4,5.7) \\
{[-4.7,6.3}\end{array}$ \\
\hline Stock returns & $(1.815 .3)$ & $(2.6,13.8)$ & $\begin{array}{l}(3.0,13.1) \\
{[2.9,13.1]}\end{array}$ & $\begin{array}{l}(3.1,12.9) \\
{[1.3,13.0]}\end{array}$ \\
\hline \multicolumn{5}{|c|}{ Longer span data series } \\
\hline GDP/Pop & $(0.2,4.5)$ & $(0.9,3.4)$ & $(1.2,2.9)$ & $(1.4,2.7)$ \\
\hline Cons/Pop & $(0.2,2.6)$ & $\begin{array}{l}(0.6,2.5) \\
{[0.6,2.6]}\end{array}$ & $\begin{array}{l}(0.8,2.4) \\
{[0.5,2.9]}\end{array}$ & $\begin{array}{l}(0.8,2.4) \\
{[0.3,3.1]}\end{array}$ \\
\hline Population & $\left(\begin{array}{l}0.5 \\
1.2\end{array}\right)$ & $(0.51 .3)$ & $(0.5,1.4)$ & $\begin{array}{l}(0.5,1.4) \\
{[0.4,1.4]}\end{array}$ \\
\hline Inflation (CPI) & $(-0.25 .9)$ & $(0.3,5.6)$ & $(0.6,5.4)$ & $(0.75 .4)$ \\
\hline Stock returns & $(0.7,13.2)$ & $(2.911 .0)$ & $(3.8,9.9)$ & $(4.2,9.5)$ \\
\hline
\end{tabular}

in the CBO model, GDP growth is $I(0)$, while the Bayes method allows values of $d$ that differ from $d=0$. The log-likelihood values plotted in Figure 2 suggest that GDP growth is plausibly characterized by a process with some low-frequency anti-persistence, and this translates into less forecast uncertainty than the CBO's I(0) model. Thus, the CBO method tends to understate forecast uncertainty because it ignores parameter uncertainty in the estimated mean and long-run variance, and to overstate forecast uncertainty because its model does not capture long-run antipersistence associated with negative values of $d$. Apparently, these two errors cancel, so that the CBO prediction interval essentially coincides with the Bayes set.

Productivity: The log-likelihood values for $d$ indicate that productivity (TFP and average labour productivity) may have somewhat greater than $I(0)$ persistence; see Figures C.3 and C.4 This translates into prediction sets that are wider than $I(0)$ sets, particularly for frequentist sets at large forecast horizons. Bayes intervals are essentially flat as the forecast horizon increases (unlike in an $I(0)$ model, where the intervals narrow), while the frequentist sets widen (the unmodified Bayes intervals systematically undercover for larger values of $d$, forcing the frequentist intervals to more heavily weigh the possibility of larger $d$ ).

Population: U.S. population growth shows considerable low-frequency variability over the 20 th century and the post-WWII period. Immigration and fertility dynamics are presumably at the source of these long swings. The low-frequency MLE of $d$ is very close to unity over both sample periods, with the $I(1) \log$-likelihood more than 7 points higher than in the $I(0)$ model. Table 5 and Supplementary Figures C.5 and C.12 show prediction intervals that widen as the forecast 
TABLE 5

Continued

(b) $90 \%$ coverage

\begin{tabular}{|c|c|c|c|c|}
\hline \multirow[t]{2}{*}{ Series } & \multicolumn{4}{|c|}{ Forecast horizon (in years) } \\
\hline & 10 & 25 & 50 & 75 \\
\hline \multicolumn{5}{|c|}{ Quarterly post-WWII series } \\
\hline GDP/Pop & $(0.3,3.7)$ & $\begin{array}{c}(0.7,3.0) \\
{[-0.3,3.0]}\end{array}$ & $\begin{array}{c}(0.8,2.8) \\
{[-0.6,2.9]}\end{array}$ & $\begin{array}{l}(0.9,2.7) \\
{[-0.93 .2]}\end{array}$ \\
\hline Cons/Pop & $(0.4,3.7)$ & $\begin{array}{c}(0.7,3.1) \\
{[0 ., 3.1]}\end{array}$ & $\begin{array}{c}(0.8,3.0) \\
{[-0.3,3.1]}\end{array}$ & $\begin{array}{c}(0.9,2.9) \\
{[-0.6,3.4]}\end{array}$ \\
\hline TF prod & $\begin{array}{l}(-0.3,2.4) \\
{[-0.6,2.4]}\end{array}$ & $\begin{array}{l}(-0.2,2.3) \\
{[-1.1,2.7]}\end{array}$ & $\begin{array}{l}(-0.1,2.3) \\
{[-1.5,3.1]}\end{array}$ & $\begin{array}{l}(-0.1,2.4) \\
{[-1.9,3.5]}\end{array}$ \\
\hline Labour prod & $\begin{array}{l}(0.0,3.2) \\
{[0.0,3.4]}\end{array}$ & $\begin{array}{c}(0.0,3.0) \\
{[-0.2,3.1]}\end{array}$ & $\begin{array}{c}(0.0,3.1) \\
{[-0.6,3.6]}\end{array}$ & $\begin{array}{l}(0.0,3.2) \\
{[-0.93 .9]}\end{array}$ \\
\hline Population & $\begin{array}{l}(0.4,1.2) \\
{[0.3,1.2]}\end{array}$ & $\begin{array}{l}(0.3,1.3) \\
{[0.1,1.5]}\end{array}$ & $\begin{array}{c}(0.1,1.5) \\
{[-0.2,1.7]}\end{array}$ & $\begin{array}{c}(0.0,1.6) \\
{[-0.5,2.0]}\end{array}$ \\
\hline Inflation (PCE) & $\begin{array}{l}(-1.1,5.0) \\
{[-1.3,5.0]}\end{array}$ & $\begin{array}{l}(-1.8,5.6) \\
{[-2.76 .1]}\end{array}$ & $\begin{array}{l}(-2.6,6.5) \\
{[-4.4,7.8]}\end{array}$ & $\begin{array}{l}(-3.2,7.1) \\
{[-5.8,9.2]}\end{array}$ \\
\hline Inflation (CPI) & $\begin{array}{l}(-1.2,6 .) \\
{[-1.4,6.0]}\end{array}$ & $\begin{array}{l}(-1.8,6.5) \\
{[-2.6,6.8]}\end{array}$ & $\begin{array}{l}(-2.5,7.2) \\
{[-4.4,8.5]}\end{array}$ & $\begin{array}{l}(-3.1,7.9) \\
{[-5.8,9.8]}\end{array}$ \\
\hline Infl. (CPI,Japan) & $\begin{array}{l}(-3.7,6.7) \\
{[-4.7,6.7]}\end{array}$ & $\begin{array}{l}(-4.7,7.7) \\
{[-6.8,8.8]}\end{array}$ & $\begin{array}{c}(-5.9,9.0) \\
{[-9.6,11.7]}\end{array}$ & $\begin{array}{c}(-6.9,10.0) \\
{[-12.0,14.0]}\end{array}$ \\
\hline Stock returns & $(-3.1,21.0)$ & $\begin{array}{l}(-2.0,20.1) \\
{[-3.8,20.1]}\end{array}$ & $\begin{array}{l}(-2.2,20.2) \\
{[-7.3,22.7]}\end{array}$ & $\begin{array}{l}(-2.520 .5) \\
{[-9.8,25.2]}\end{array}$ \\
\hline \multicolumn{5}{|c|}{ Longer span data series } \\
\hline GDP/Pop & $\begin{array}{l}(-1.4,6.2) \\
{[-1.8,6.5]}\end{array}$ & $(-0.1,4.3)$ & $(0.4,3.6)$ & $(0.7,3.3)$ \\
\hline Cons/Pop & $\begin{array}{l}(-0.7,3.5) \\
{[-0.7,4.0]}\end{array}$ & $\begin{array}{l}(-0.3,3.1) \\
{[-0.6,3.5]}\end{array}$ & $\begin{array}{c}(-0.2,3.0) \\
{[-0.9,3.9]}\end{array}$ & $\begin{array}{l}(-0.1,3.0) \\
{[-1.3,4.2]}\end{array}$ \\
\hline Population & $(0.3,1.5)$ & $\begin{array}{l}(0.2,1.6) \\
{[0.1,1.6]}\end{array}$ & $\begin{array}{c}(0.1,1.7) \\
{[-0.2,1.8]}\end{array}$ & $\begin{array}{c}(0.0,1.8) \\
{[-0.4,2.0]}\end{array}$ \\
\hline Inflation (CPI) & $(-2.6,8.2)$ & $(-2.3,7.8)$ & $\begin{array}{l}(-2.4,8.0) \\
{[-2.9,8.1]}\end{array}$ & $\begin{array}{l}(-2.7,8.3) \\
{[-3.7,9.0]}\end{array}$ \\
\hline Stock returns & $\begin{array}{l}(-3.9,18.4) \\
{[-5.7,18.4]}\end{array}$ & $(-0.215 .2)$ & $(1.1,13.9)$ & $\begin{array}{l}(1.613 .4) \\
{[1.2,13.4]}\end{array}$ \\
\hline
\end{tabular}

Notes: This table shows the $67 \%$ and $90 \%$ prediction sets for forecast horizons, $h=10,25,50$, and 75 years. The $A^{\text {Bayes }}$ sets are shown in parentheses and are based on the $I(d)$ model with uniform prior for $-0.4 \leqslant d \leqslant 1.0$. The $A^{M N}$ sets are shown in brackets, and are omitted if they coincide with the $A^{\text {Bayes }}$ sets. By construction the $A^{M N}$ sets control asymptotic coverage in the $b c d$-model with $-0.4 \leqslant d \leqslant 1.0$, and $b$ and $c$ unrestricted.

horizon increases, a natural characteristic of $I(1)$ predictive densities. There is little difference in the sets constructed using the post-WWII samples and long-samples.

Inflation: As discussed above, the inflation process is characterized by more than $I(0)$ persistence, and this is reflected in the prediction sets in two ways. First, they are not centred at the sample mean of the series, but rather at a level dictated by the values near the end of sample period, and secondly, the prediction sets widen with the forecast horizon. The prediction intervals indicate considerable uncertainty in inflation even at relatively short horizons; this is true for both Bayes and frequentist sets. For example, while the 10-year 67\% Bayes prediction set for U.S. CPI inflation is $(0.4,4.5)$, the $90 \%$ set widens to $(-1.2,6.0)$.

These predictions sets may strike some readers as too large, but it is instructive to consider the history of Japan where the 10-year moving average of CPI inflation was less than zero from 2003 through 2013 (Supplementary Figure C.8). Moreover, they are in line with predictive densities derived from asset prices. For example, Kitsul and Wright 2013) use CPI-based derivatives to compute market-based risk-neutral predictive densities for 10 -year-ahead average values of inflation. They find deflation (average inflation less than $0 \%$ ) probabilities that averaged 
TABLE 6

Prediction sets for various values of $q$, 25-year horizon

\begin{tabular}{|c|c|c|c|c|c|c|}
\hline & \multicolumn{3}{|c|}{$67 \%$} & \multicolumn{3}{|c|}{$90 \%$} \\
\hline & $q=6$ & $q=12$ & $q=24$ & $q=6$ & $q=12$ & $q=24$ \\
\hline \multicolumn{7}{|c|}{ Quarterly post-WWII data } \\
\hline \multirow[t]{2}{*}{ GDP/Pop } & $(0.6,2.3)$ & $(1.3,2.6)$ & $(1.5,2.6)$ & $(-0.1,2.7)$ & $(0.7,3.0)$ & $(0.9,3.0)$ \\
\hline & {$[0.2,2.3]$} & {$[1.2,2.6]$} & & {$[-0.8,2.7]$} & {$[-0.3,3.0]$} & {$[0.0,3.0]$} \\
\hline \multirow{2}{*}{ Cons/Pop } & $(0.9,2.6)$ & $(1.4,2.7)$ & $(1.4,2.6)$ & $(0.0,3.1)$ & $(0.7,3.1)$ & $(0.7,3.1)$ \\
\hline & & & & {$[-0.5,3.1]$} & {$[0.0,3.1]$} & {$[0.0,3.1]$} \\
\hline \multirow[t]{2}{*}{ TF prod } & $(0.1,1.6)$ & $(0.5,1.8)$ & $(0.6,1.8)$ & $(-0.5,2.1)$ & $(-0.2,2.3)$ & $(0.1,2.2)$ \\
\hline & {$[-0.2,1.6]$} & {$[-0.2,1.9]$} & {$[-0.2,2.0]$} & {$[-1.1,2.3]$} & {$[-1.1,2.7]$} & {$[-1.0,2.7]$} \\
\hline \multirow[t]{2}{*}{ Labour prod } & $(1.0,2.6)$ & $(1.0,2.6)$ & $(1.2,2.5)$ & $(0.2,3.1)$ & $(0.0,3.0)$ & $(0.5,3.0)$ \\
\hline & & & {$[0.9,2.5]$} & {$[-0.4,3.1]$} & {$[-0.2,3.1]$} & {$[-0.2,3.2]$} \\
\hline \multirow{2}{*}{ Population } & $(0.5,1.2)$ & $(0.5,1.1)$ & $(0.5,1.1)$ & $(0.3,1.5)$ & $(0.3,1.3)$ & $(0.3,1.3)$ \\
\hline & {$[0.4,1.3]$} & {$[0.4,1.2]$} & {$[0.4,1.1]$} & {$[0.0,1.6]$} & {$[0.1,1.5]$} & {$[0.1,1.4]$} \\
\hline \multirow[t]{2}{*}{ Inflation (PCE) } & $(0.4,4.7)$ & $(0.0,4.1)$ & $(-0.2,3.8)$ & $(-1.7,6.3)$ & $(-1.8,5.6)$ & $(-2.0,5.3)$ \\
\hline & & & & {$[-3.1,6.5]$} & {$\left[\begin{array}{lll}-2.7 & 6.1\end{array}\right]$} & {$[-2.6,5.4]$} \\
\hline \multirow[t]{2}{*}{ Inflation (CPI) } & $(0.6,5.3)$ & $(0.3,4.8)$ & $(0.0,4.5)$ & $(-1.8,7.0)$ & $(-1.8,6.5)$ & $(-2.1,6.2)$ \\
\hline & & & & {$[-3.3,7.2]$} & {$[-2.6,6.8]$} & {$[-2.5,6.2]$} \\
\hline \multirow{2}{*}{ Infl. (CPI, Japan) } & $(-1.6,5.2)$ & $(-1.7,4.9)$ & $(-1.3,4.9)$ & $(-4.78 .0)$ & $(-4.7,7.7)$ & $(-3.9,7.7)$ \\
\hline & {$[-3.7,5.2]$} & {$[-2.4,4.9]$} & & {$[-8.2,9.7]$} & {$[-6.8,8.8]$} & {$[-5.5,7.9]$} \\
\hline \multirow[t]{2}{*}{ Stock returns } & $(2.0,13.1)$ & $(2.6,13.8)$ & $(2.7,12.8)$ & $(-3.1,18.8)$ & $(-2.0,20.1)$ & $(-0.616 .4)$ \\
\hline & & & & & {$[-3.8,20.1]$} & {$\left[\begin{array}{lll}-9.0 & 24.0\end{array}\right]$} \\
\hline \multicolumn{7}{|c|}{ Longer span data series } \\
\hline GDP/Pop & $(0.8,2.9)$ & $(0.9,3.4)$ & $(0.8,3.2)$ & $(-0.2,3.8)$ & $(-0.1,4.3)$ & $(-0.3,4.1)$ \\
\hline \multirow[t]{2}{*}{ Cons/Pop } & $(0.7,2.5)$ & $(0.6,2.5)$ & $(0.9,2.6)$ & $(-0.1,3.3)$ & $(-0.3,3.1)$ & $(0.2,3.3)$ \\
\hline & & {$[0.6,2.6]$} & {$[0.9,2.7]$} & {$[-0.3,3.4]$} & {$[-0.6,3.5]$} & {$[-0.2,3.7]$} \\
\hline \multirow[t]{2}{*}{ Population } & $(0.8,1.6)$ & $(0.5,1.3)$ & $(0.5,1.3)$ & $(0.4,1.9)$ & $(0.2,1.6)$ & $(0.2,1.5)$ \\
\hline & & & & {$[0.3,2.0]$} & {$[0.1,1.6]$} & {$[0.1,1.5]$} \\
\hline \multirow[t]{2}{*}{ Inflation (CPI) } & $(0.3,6.1)$ & $(0.3,5.6)$ & $(0.8,5.3)$ & $(-2.3,8.7)$ & $(-2.3,7.8)$ & $(-1.1,7.0)$ \\
\hline & & & & & & {$[-1.6,7.5]$} \\
\hline Stock returns & $(2.0,11.5)$ & $(2.9,11.0)$ & $(2.8,9.2)$ & $(-2.4,15.8)$ & $(-0.2,15.2)$ & $(0.4,11.8)$ \\
\hline
\end{tabular}

Notes: $A^{\text {Bayes }}$ sets are shown in parentheses and $A^{M N}$ sets are shown in brackets when they differ from Bayes sets. See notes to Table 5 for additional information.

approximately $15 \%$ over 2011 and "high inflation" (average inflation greater than $4 \%$ ) of $30 \% 15$ The corresponding probabilities computed from the Bayes predictive density constructed using the post-WWII data are $11 \%$ for deflation and $28 \%$ for high inflation.

Stock Returns: Post-WWII real stock returns exhibit slightly more persistence than what is implied by the $I(0)$ model, and this translates into prediction sets that are wider than that implied by the $I(0)$ model. For example, at the 25 -year horizon, the $67 \%-I(0)$ prediction set (from Supplementary Figure C.9) is $(3.4,11.2)$ while the corresponding Bayes and MN prediction sets (from Table 7) are $(1.8,15.3)$. The longer-span data suggest somewhat less persistence (the MLE of $\mathrm{d}$ is -0.2 for the 1926-2014 sample) yielding Bayes and frequentist prediction intervals that are somewhat narrower than those constructed using the post-WWII data.

Pastor and Stambaugh 2012) survey the large literature on long-run stock return volatility and construct Bayes predictive densities using models that allow for potentially persistent components in returns. While their results rely on more parametric models than ours - they use all frequencies and exact Gaussian likelihoods-our empirical conclusions are similar. Using our notation,

15. See Kitsul and Wrigh 2013, Figures 3 and 4. Fleckenstein et al. 2013 estimate somewhat lower probabilites for deflation, but similar probabilities for inflation exceeding 4\%. (See their Figures 4 and 5.) For a related calculation, see Figure 3 in Hilsher et al. 2014. 
Pastor and Stambaugh 2012) are concerned with the behaviour of the variance of $\sqrt{h} \bar{x}_{T+1: T+h}$ and how this variance changes with the forecast horizon $h$. If the variance of $\sqrt{h} \bar{x}_{T+1: T+h}$ is unchanged as $h$ increases, and if the predictive density is Gaussian, then the width of prediction intervals for $\bar{x}_{T+1: T+h}$ will be proportional to $h^{-1 / 2}$. Pastor and Stambaugh find that the variance of $\sqrt{h} \bar{x}_{T+1: T+h}$ is not constant, but rather increases with $h$. Consistent with this, we find Bayes prediction sets that narrow as $h$ increases, but more slowly than $h^{-1 / 2}$.

Results for different values of $q$ : As discussed in Section 4 the choice of $q=12$ involved an efficiency/robustness trade-off, where a larger value of $q$ results in more information about the scale and shape parameter, but potential misspecification because the higher-frequency spectrum may not be well described by the same model and parameter. It is, therefore, interesting to see how the prediction sets vary with $q$, and this is reported in Table 6 which shows the $67 \%$ and $90 \%$ prediction sets for the 25-year-ahead forecasts for $q=6,12$, and 24 . Looking across all of the entries, the prediction sets behave roughly as expected, in the sense that they remain centred at roughly the same value but tend to narrow as $q$ increases. For example, averaging across the 14 series, the $67 \% \mathrm{MN}$ prediction set is $11 \%$ narrower using $q=24$ than with $q=12$ broadly consistent the results discussed in Section 4

\section{CONCLUSIONS}

This article has considered the problem of quantifying uncertainty about long-run predictions using prediction sets that contain the realized future value of a variable of interest with prespecified probability. The long-run nature of the problem both simplifies and complicates the problem relative to short-run predictions. The problem is simplified because of our focus on forecasting long-run averages using a relatively small number of (low-frequency) weighted averages of the sample data. As we show, these averages conveniently have an approximate joint normal distribution under fairly general conditions. However, the prediction problem is complicated because the covariance matrix of the limiting normal distribution depends on the shape of the spectrum over very low frequencies, and there is limited sample information about this shape. Uncertainty about the low-frequency characteristics of the stochastic process is then an important component of the uncertainty about long-run predictions.

We proposed a flexible parametric model (the $b c d$-model) to characterize the shape of the spectrum at low frequencies. Uncertainty about the shape then becomes equivalent to uncertainty about the values of the $b c d$-parameters. Incorporating this parameter uncertainty into prediction uncertainty is straightforward in a Bayesian framework, and we provide the details in the context of the long-run prediction problem. However, because of the paucity of sample information about these long-run parameters, the resulting Bayes prediction sets may depend importantly on the specifics of the prior. This motivates us to robustify the Bayes sets by enlarging them so that, by construction, they control coverage uniformly over all values of the $b c d$-parameters. We construct minimum expected length frequentist prediction sets using an approximate "least favourable distribution" for the parameters, and we generalize these to conditionally sensible frequentist prediction sets using ideas from Müller and Norets 2012).

We apply these methods and construct prediction sets for nine macroeconomic time series for forecast horizons of up to 75 years. In general, we found that for many series, the prediction sets are wider than those that one obtains from the $I(0)$ model, but narrower than one would obtain from, say, the $I(1)$ model. From a statistical point of view, this underlines the importance of modelling the spectral shape at low frequencies in a flexible manner. Substantively, it demonstrates that even after accounting for a wide variety of potential long-run instabilities and dependencies, it is still possible to make informative probability statements about (very) long-run forecasts. 
While the analysis presented in this article accommodates a wide range of low-frequency persistence patterns, it was not designed to directly accommodate large breaks in volatility such as those evident in the pre- and post-WWII U.S. GDP growth rate data. In principle, it is possible to explicitly account for non-negligible non-stationarities in the volatility process by postulating a stochastic process for the volatility path, and integrating out this additional source of uncertainty (similar to the approach of Müller and Watson, 2013 in their Section 3.3, for instance).

Also, our analysis has been univariate in the sense that we have constructed predictions sets for a scalar random variable $\bar{x}_{T+1: T+h}$ using sample values of $x_{t}$. However, answers to some questions require multivariate prediction sets. The statistical theory discussed and developed in Section 3 carries over directly to multivariate settings. That said, there are important practical obstacles to constructing multivariate prediction sets. These obstacles include finding a convenient, but flexible, parameterization of the multivariate local-to-zero spectrum, constructing accurate approximations to least favourable distributions with high-dimensional $\theta$, and computing accurate approximations to the density of relevant invariants. Overcoming these obstacles is left to future research.

\section{APPENDIX}

\section{A. A CENTRAL LIMIT THEOREM}

Theorem 1. Let $\Delta x_{T, t}=\sum_{s=-\infty}^{\infty} c_{T, s} \varepsilon_{t-s}$. Suppose that

(i) $\left\{\varepsilon_{t}, \mathcal{F}_{t}\right\}$ is a martingale difference sequence with $E\left(\varepsilon_{t}^{2}\right)=1$, $\sup _{t} E\left(\left|\varepsilon_{t}\right|^{2+\delta}\right)<\infty$ for some $\delta>0$, and

$$
E\left(\varepsilon_{t}^{2}-1 \mid \mathcal{F}_{t-m}\right) \leq \xi_{m}
$$

for some sequence $\xi_{m} \rightarrow 0$;

(ii) for every $\epsilon>0$ the exists an integer $L_{\epsilon}>0$ such that $\limsup _{T \rightarrow \infty} T^{-1} \sum_{l=L_{\epsilon} T+1}^{\infty}\left(T \sup _{|s| \geq l}\left|c_{T, s}\right|\right)^{2}<\epsilon$;

(iii) $\sum_{s=-\infty}^{\infty} c_{T, s}^{2}<\infty$ (but not necessarily uniformly in $T$ ). The spectral density of $\Delta x_{T, t}$ thus exists; denote it by $F_{T}:[-\pi, \pi] \mapsto \mathbb{R}$

(iii.a) there exists a function $S: \mathbb{R} \mapsto \mathbb{R}$ such that $\omega \mapsto \omega^{2} S(\omega)$ is integrable, and for all fixed $K$,

$$
\int_{0}^{K}\left|F_{T}\left(\frac{\omega}{T}\right)-\omega^{2} S(\omega)\right| d \omega \rightarrow 0 ;
$$

(iii.b) for every diverging sequence $K_{T} \rightarrow \infty$

$$
T^{-3} \int_{K_{T} / T}^{\pi} F_{T}(\lambda) \lambda^{-4} d \lambda=\int_{K_{T}}^{\pi T} F_{T}(\omega / T) \omega^{-4} d \omega \rightarrow 0 ;
$$

(iii.c)

$$
T^{-3 / 2} \int_{1 / T}^{\pi} F_{T}(\lambda)^{1 / 2} \lambda^{-2} d \lambda=T^{-1 / 2} \int_{1}^{\pi T} F_{T}(\omega / T)^{1 / 2} \omega^{-2} d \omega \rightarrow 0 ;
$$

(iv) for some fixed integer $H$, the function $g:[0, H] \mapsto \mathbb{R}$ is of bounded variation and satisfies $\int_{0}^{H} g(s) d s=0$.

Then

$$
T^{-1 / 2} \int_{0}^{H} g(s) x_{T,\lfloor s T\rfloor+1} d s \Rightarrow \mathcal{N}\left(0, \int_{-\infty}^{\infty} S(\omega)\left|\int_{0}^{H} e^{-i \omega s} g(s) d s\right|^{2} d \omega\right)
$$

where $x_{T, t}=\sum_{s=1}^{t} \Delta x_{T, s}$

Remarks. Note that the linear process $\Delta x_{T, t}$ is not restricted to be causal. The m.d.s. structure of the driving errors $\varepsilon_{t}$ in assumption (i) allows for some departures from strict stationarity. It also accommodates conditional heteroscedasticity, with the second-order dependence limited by the mixingale condition A.1.

The linear coefficients $c_{T, s}$ are scaled by the sample size $T$ such that the convergence A.5 holds with the same scaling factor $T^{-1 / 2}$ across various types of persistence, such as $I(0)$ and $I(1)$ models. See below for examples. Given our interest in scale equivariant prediction sets, this scale normalization is without loss of generality.

Since for any fixed $K, \sup _{0 \leq \omega \leq K}\left|T^{-2} \frac{\omega^{2}}{\left|1-e^{-i \omega / T}\right|^{2}}-1\right| \rightarrow 0$, assumption (iii.a) is equivalent to equation 5 (with $\kappa=3 / 2$ ). 
To better understand the role of assumptions (ii) and (iii), consider some leading examples. Suppose first that $\Delta x_{T, t}$ is causal and weakly dependent with exponentially decaying $c_{T, s},\left|c_{T, s}\right| \leq C_{0} e^{-C_{1} s}$ for some $C_{0}, C_{1}>0$, as would arise in causal and invertible ARMA models of any fixed and finite order. Then $T^{-1} \sum_{l=L T+1}^{\infty}\left(T \sup _{|s|>l}\left|c_{T, s}\right|\right)^{2} \rightarrow 0$ for any $L>0, \omega^{2} S(\omega)$ is constant and equal to $(2 \pi)^{-1}$ times the long-run variance of $\Delta x_{T, t}$, and conditions A.3 and A.4 hold, since $F_{T}$ is bounded, $\int_{K_{T}}^{\infty} \omega^{-4} d \omega \rightarrow 0$ for any $K_{T} \rightarrow \infty$ and $\int_{1}^{\infty} \omega^{-2} d \omega<\infty$.

Secondly, suppose $\Delta x_{T, t}$ is fractionally integrated with parameter $d \in(-1 / 2,1 / 2)$ (corresponding to $x_{T, t}$ being fractionally integrated of order $\left.d+1\right)$. With $\Delta x_{T, t}$ scaled by $T^{-d}, c_{T, s} \approx C_{0} T^{-d} s^{d-1}$, so that $T^{-1} \sum_{l=L T+1}^{\infty}\left(T \sup _{|s| \geq l}\left|c_{T, s}\right|\right)^{2} \rightarrow \int_{L}^{\infty} s^{2 d-2} d s$, which can be made arbitrarily small by choosing $L$ large. Further, for $\lambda$ close to zero, $F_{T}(\lambda) \approx(2 \pi)^{-1} C_{0}^{2}(\lambda T)^{-2 d}$, so that $\omega^{2} S(\omega)=(2 \pi)^{-1} C_{0}^{2} \omega^{-2 d}$, and conditions A.3 and A.4 are seen to hold under weak assumptions about higher frequency properties of $\Delta x_{T, t}$. For instance, even integrable poles in $F_{T}$ at frequencies other than zero can be accommodated.

Thirdly, suppose $x_{T, t}$ is an AR(1) process with local-to-unity coefficient $\rho_{T}=1-c / T$ and unit innovation variance. Then $c_{T, 0}=1$ and $c_{T, s}=-\left(1-\rho_{T}\right) \rho_{T}^{s}, s>0$. Thus, $T^{-1} \sum_{l=L T+1}^{\infty}\left(T \sup _{|s| \geq l}\left|c_{T, s}\right|\right)^{2} \rightarrow c^{2} \int_{L}^{\infty} e^{-2 c s} d s$, which can be made arbitrarily small by choosing $L$ large. Further, $F_{T}(\lambda)=(2 \pi)^{-1}\left|1-e^{-\mathfrak{i} \lambda}\right|^{2} /\left|1-\rho_{T} e^{-\mathfrak{i} \lambda}\right|^{2}$, which is seen to satisfy equation A.2 with $S(\omega)=(2 \pi)^{-1}\left(\omega^{2}+c^{2}\right)^{-1}$. Conditions A.3 and A.4 also hold in this example, since $F_{T}(\lambda) \leq 1$.

As a final example, suppose $\Delta x_{T, t}=T \varepsilon_{t}-T \varepsilon_{t-1}$ (inducing $x_{T, t}$ to be i.i.d. conditional on $\varepsilon_{0}$, with a scaling such that $F_{T}(\lambda)$ is $O_{p}(1)$ for $\left.\lambda=O\left(T^{-1}\right)\right)$. Here, $F_{T}(\lambda)=(2 \pi)^{-1} T^{2}\left|1-e^{-i \lambda}\right|^{2}=(2 \pi)^{-1} 4 T^{2} \sin (\lambda / 2)^{2}$, so that $S(\omega)=(2 \pi)^{-1}$, and condition A.3 evaluates to $4(2 \pi)^{-1} \int_{K_{T}}^{\pi T} T^{2} \sin (\omega / 2 T)^{2} \omega^{-4} d \omega \leq(2 \pi)^{-1} \int_{K_{T}}^{\pi T} \omega^{-2} d \omega \rightarrow 0$, and condition A.4 to $2(2 \pi)^{-1 / 2} T^{-1 / 2} \int_{1}^{\pi T} T \sin (\omega / 2 T) \omega^{-2} d \omega \leq(2 \pi)^{-1 / 2} T^{-1 / 2} \int_{1}^{\pi T} \omega^{-1} d \omega \rightarrow 0$, where the inequalities follow from $\sin (\lambda) \leq \lambda$ for all $\lambda \geq 0$.

The number $H$ is assumed to be an integer to ease notation. Note that a constant $g$ would not satisfy assumption (iv), as it does not integrate to zero, but all functions of interest in the context of this article do. The implication of Theorem 1 that is of interest for Section 3 follows from the following Corollary.

Corollary 1. For some $0<r<H-1$, let $g_{q+1}:[0, H] \mapsto \mathbb{R}$ equal $g_{q+1}(s)=-\mathbf{1}[0 \leq s \leq 1]+r^{-1} \mathbf{1}[1<s \leq 1+r]$ and let $g_{j}:[0, H] \mapsto \mathbb{R}$ equal to $g_{j}(s)=\mathbf{1}[s \leq 1] \sqrt{2} \cos (\pi j s)$ for $j=1, \ldots, q$. Under the assumptions of Theorem $1(i)-(i i i)$,

$$
T^{-1 / 2} \int_{0}^{H}\left[\begin{array}{c}
g_{1}(s) \\
\vdots \\
g_{q}(s) \\
g_{q+1}(s)
\end{array}\right] x_{T,\lfloor s T\rfloor+1} d s \Rightarrow \mathcal{N}(0, \Sigma)
$$

where $\Sigma_{j, k}=\int_{-\infty}^{\infty} S(\omega)\left(\int_{0}^{H} e^{-i \omega s} g_{j}(s) d s\right)\left(\int_{0}^{H} e^{i \omega s} g_{k}(s) d s\right) d \omega$ for $j, k=1, \ldots, q+1$.

Proof. Follows from Theorem 1 and the Cramer-Wold device via

$$
\left|\int_{0}^{H} e^{-i \omega s}\left(\sum_{j=1}^{q+1} \lambda_{j} g_{j}(s)\right) d s\right|^{2}=\sum_{j, k=1}^{q+1} \lambda_{j} \lambda_{k}\left(\int_{0}^{H} e^{-i \omega s} g_{j}(s) d s\right)\left(\int_{0}^{H} e^{i \omega s} g_{k}(s) d s\right)
$$

since $g(s)=\sum_{j=1}^{q+1} \lambda_{j} g_{j}(s)$ clearly satisfies the assumption in Theorem 1 (iv). $\quad \|$

\section{B. DENSITY OF $\left(\mathbf{X}^{S}, Y^{S}\right)$ AND RELATED RESULTS}

Let $\mathbf{Z}=\left(\mathbf{X}^{\prime}, Y\right)^{\prime}$ and $U=\sqrt{\mathbf{X}^{\prime} \mathbf{X}}$. Write $\mu_{l}$ for Lebesgue measure on $\mathbb{R}^{l}$, and $v_{q}$ for the surface measure of a $q$-dimensional unit sphere. For $\mathbf{x} \in \mathbb{R}^{q}$, let $\mathbf{x}=\mathbf{x}^{s} u$, where $\mathbf{x}^{s}$ is a point on the surface of a $q$-dimensional unit sphere, and $u \in \mathbb{R}^{+}$. By Theorem 2.1.13 of Muirhead 1982), $d \mu_{q}(\mathbf{x})=u^{q-1} d v_{q}\left(\mathbf{x}^{s}\right) d \mu_{1}(u)$. Further, for $y \in \mathbb{R}$, consider the change of variable $y=y^{s} u$ with $u \in \mathbb{R}^{+}$and $y^{s} \in \mathbb{R}$, so that $d \mu_{1}(y)=u d \mu_{1}\left(y^{s}\right)$. We thus can write the joint density of $\left(\mathbf{X}^{s}, Y^{s}, U\right)$ with respect to $v_{q} \times \mu_{1} \times \mu_{1}$ as

$$
(2 \pi)^{-(q+1) / 2}|\Sigma|^{-1 / 2} \exp \left[-\frac{1}{2}\left(\begin{array}{c}
\mathbf{x}^{s} u \\
y^{s} u
\end{array}\right)^{\prime} \Sigma^{-1}\left(\begin{array}{c}
\mathbf{x}^{s} u \\
y^{s} u
\end{array}\right)\right] u^{q}
$$


and the marginal density of $\mathbf{Z}^{s}=\left(\mathbf{X}^{s^{\prime}}, Y^{s}\right)^{\prime}$ with respect to $v_{q} \times \mu_{1}$ is

$$
\begin{aligned}
f_{\mathbf{Z}^{s}}\left(\mathbf{z}^{s}\right) & =(2 \pi)^{-(q+1) / 2}|\Sigma|^{-1 / 2} \int_{0}^{\infty} u^{q} \exp \left[-\frac{1}{2} u^{2}\left(\mathbf{z}^{s^{\prime}} \Sigma^{-1} \mathbf{z}^{s}\right)\right] d \mu_{1}(u) \\
& =(2 \pi)^{-(q+1) / 2}|\Sigma|^{-1 / 2} \frac{1}{2} \int_{0}^{\infty} t^{(q-1) / 2} \exp \left[-\frac{1}{2} t\left(\mathbf{z}^{s \prime} \Sigma^{-1} \mathbf{z}^{s}\right)\right] d \mu_{1}(t) \\
& =(2 \pi)^{-(q+1) / 2}|\Sigma|^{-1 / 2} \frac{1}{2} \Gamma\left(\frac{q+1}{2}\right) 2^{(q+1) / 2}\left(\mathbf{z}^{s^{\prime}} \Sigma^{-1} \mathbf{z}^{s}\right)^{-(q+1) / 2} \\
& =\frac{1}{2} \pi^{-(q+1) / 2}|\Sigma|^{-1 / 2} \Gamma\left(\frac{q+1}{2}\right)\left(\mathbf{z}^{s^{\prime}} \Sigma^{-1} \mathbf{z}^{s}\right)^{-(q+1) / 2}
\end{aligned}
$$

where the second equality follows from the form of the Gamma density function, and $\Gamma$ denotes the gamma function. The implied marginal density of $\mathbf{X}^{s}$ is

$$
f_{\mathbf{X}^{s}}\left(\mathbf{x}^{s}\right)=\frac{1}{2} \pi^{-(q) / 2}\left|\Sigma_{X X}\right|^{-1 / 2} \Gamma\left(\frac{q}{2}\right)\left(\mathbf{x}^{s /} \Sigma_{X X}^{-1} \mathbf{x}^{s}\right)^{-q / 2} .
$$

Similarly, with $g\left(\mathbf{x}^{s}\right)=E\left[\sqrt{\mathbf{X}^{\prime} \mathbf{X}} \mid \mathbf{X}^{s}=\mathbf{x}^{s}\right]$, we obtain

$$
\begin{aligned}
f_{\mathbf{X}^{s}}\left(\mathbf{x}^{s}\right) g\left(\mathbf{x}^{s}\right) & =\int_{0}^{\infty} u f_{\left(\mathbf{X}^{s}, U\right)}\left(\mathbf{x}^{s}, u\right) d \mu_{1}(u) \\
& =(2 \pi)^{-q / 2}\left|\Sigma_{X X}\right|^{-1 / 2} \int_{0}^{\infty} u^{q} \exp \left[-\frac{1}{2} u^{2}\left(\mathbf{x}^{s \prime} \Sigma_{X X}^{-1} \mathbf{x}^{s}\right)\right] d \mu_{1}(u) \\
& =2^{-1 / 2} \pi^{-q / 2}\left|\Sigma_{X X}\right|^{-1 / 2} \Gamma\left(\frac{q+1}{2}\right)\left(\mathbf{x}^{s \prime} \Sigma_{X X}^{-1} \mathbf{x}^{s}\right)^{-(q+1) / 2}
\end{aligned}
$$

Finally, from equation [6, $\tilde{Y}=Y-\Sigma_{Y X} \Sigma_{X X}^{-1} \mathbf{X} \sim \mathcal{N}\left(0, \Sigma_{Y Y}-\Sigma_{Y X} \Sigma_{X X}^{-1} \Sigma_{X Y}\right)$ and $\mathbf{X}$ are independent normal random variables. Also, using well-known properties of a multivariate standard normal distribution, $\mathbf{X}^{\prime} \Sigma_{X X}^{-1} \mathbf{X} \sim \chi_{q}^{2}$ is independent of $\tilde{\mathbf{X}}^{s}=\Sigma_{X X}^{-1 / 2} \mathbf{X} / \sqrt{\mathbf{X}^{\prime} \Sigma_{X X}^{-1} \mathbf{X}}$. Since $\mathbf{X}^{s}$ is a one-to-one transformation of $\tilde{\mathbf{X}}^{s}$, we thus obtain

$$
\frac{\tilde{Y}}{\sqrt{\mathbf{X}^{\prime} \Sigma_{X X}^{-1} \mathbf{X} / q} \sqrt{\Sigma_{Y Y}-\Sigma_{Y X} \Sigma_{X X}^{-1} \Sigma_{X Y}}} \mid \mathbf{X}^{s} \sim \text { Student- } t^{q}
$$

and the result 8 follows by dividing the numerator and denominator by $\sqrt{\mathbf{X}^{\prime} \mathbf{X}}$.

\section{APPROXIMATE LEAST FAVOURABLE DISTRIBUTIONS}

In practice, it will not be possible to compute a least favourable distribution $\Lambda^{\dagger}$ that perfectly solves the program 14,16 . To make further progress, we follow Elliott et al. 2015) (EMW in the following), and first formally state a lower bound on equation 14, and then define an $\operatorname{ALFD} \Lambda^{*}$ that solves 10 within a tolerance of $\epsilon$.

To ease notation, write $V_{W}(A)=\int V_{\theta}(A) d W(\theta)$ and $C_{\theta}(A)=P_{\theta}\left(Y^{s} \in A\left(\mathbf{X}^{s}\right)\right)$. Also, we make the dependence of the set 18 on cv explicit by writing

$$
A_{\Lambda, \mathrm{cv}}\left(\mathbf{x}^{s}\right)=\left\{y^{s}: \frac{\int f_{\left(Y^{s}, \mathbf{X}^{s}\right) \mid \theta}\left(y^{s}, \mathbf{x}^{s}\right) d \Lambda(\theta)}{\int g_{\theta}\left(\mathbf{x}^{s}\right) f_{\mathbf{X}^{s} \mid \theta}\left(\mathbf{x}^{s}\right) d W(\theta)}>\mathrm{cv}\right\} .
$$

We begin by proving the optimality of the set $A_{\Lambda, \mathrm{cv}}$ in the problem $\min _{A} V_{W}(A)$ subject to $\int C_{\theta}(A) d \Lambda(\theta)=1-\alpha$.

Lemma 1. Let $A_{\Lambda, \mathrm{cv}}$ be such that $\int C_{\theta}\left(A_{\Lambda, \mathrm{cv}}\right) d \Lambda(\theta)=1-\alpha$. Then $A_{\Lambda, \mathrm{cv}}$ solves $\min _{A} V_{W}(A)$ subject to $\int C_{\theta}(A) d \Lambda(\theta) \geq$ $1-\alpha$.

Proof. Note that any $A$ is equivalently characterized by the test-function $\varphi: \mathbb{R}^{q} \times \mathbb{R} \mapsto\{0,1\}$ defined via $\varphi\left(y^{s}, \mathbf{x}^{s}\right)=$ $\mathbf{1}\left[y^{s} \in A\left(\mathbf{x}^{s}\right)\right]$. In this notation, $V_{W}(A)=\iiint g_{\theta}\left(\mathbf{x}^{s}\right) f_{\mathbf{X}^{s} \mid \theta}\left(\mathbf{x}^{s}\right) \varphi\left(y^{s}, \mathbf{x}^{s}\right) d v_{q}\left(\mathbf{x}^{s}\right) d \mu_{1}\left(y^{s}\right) d W(\theta)=\int \varphi\left(\mathbf{z}^{s}\right) f_{1}\left(\mathbf{z}^{s}\right) d \lambda_{q, 1}\left(\mathbf{z}^{s}\right)$, and $\int C_{\theta}(A) d \Lambda(\theta)=\iiint f_{\mathbf{Z}^{s} \mid \theta}\left(\mathbf{x}^{s}, y^{s}\right) \varphi\left(y^{s}, \mathbf{x}^{s}\right) d v_{q}\left(\mathbf{x}^{s}\right) d \mu_{1}\left(y^{s}\right) d \Lambda(\theta)=\int \varphi\left(\mathbf{z}^{s}\right) f_{0}\left(\mathbf{z}^{s}\right) d \lambda_{q, 1}\left(\mathbf{z}^{s}\right)$, where $d \lambda_{q, 1}\left(\mathbf{z}^{s}\right)=d v_{q}\left(\mathbf{x}^{s}\right) \times$ $d \mu_{1}\left(y^{s}\right), f_{1}\left(\mathbf{z}^{s}\right)=\int g_{\theta}\left(\mathbf{x}^{s}\right) f_{\mathbf{X}^{s} \mid \theta}\left(\mathbf{x}^{s}\right) d W(\theta)$ and $f_{0}\left(\mathbf{z}^{s}\right)=\int f_{\mathbf{Z}^{s} \mid \theta}\left(\mathbf{z}^{s}\right) d \Lambda(\theta)$. Thus, the problem is equivalent to the problem of finding the best test that rejects (that is $\varphi=1$ ) with probability at least $1-\alpha$ when the "density" of $\mathbf{Z}^{s}$ is $f_{0}$, and minimizes the rejection probability when the "density" of $\mathbf{Z}^{s}$ is $f_{1}$. These densities do not necessarily integrate to one, but the solution still has to be of the Neyman-Pearson form 18, as can be seen by the very argument that proves the Neyman-Pearson 
Lemma: Set $\varphi^{*}\left(y^{s}, \mathbf{x}^{s}\right)=\mathbf{1}\left[y^{s} \in A_{\Lambda, \mathrm{cv}}\left(\mathbf{x}^{s}\right)\right]$ and $\varphi\left(y^{s}, \mathbf{x}^{s}\right)=\mathbf{1}\left[y^{s} \in A\left(\mathbf{x}^{s}\right)\right]$ for some $A$ that satisfies $\int C_{\theta}(A) d \Lambda(\theta) \geq 1-\alpha$. Then $\int \varphi f_{0} d \lambda_{q, 1} \geq 1-\alpha$ (we drop $\mathbf{z}^{s}$ as the dummy variable of integration for notational convenience), and

$$
\begin{aligned}
0 & \leq \int\left(\varphi^{*}-\varphi\right)\left(f_{0}-\operatorname{cv} f_{1}\right) d \lambda_{q, 1} \\
& \leq \operatorname{cv}\left(\int \varphi f_{1} d \lambda_{q, 1}-\int \varphi^{*} f_{1} d \lambda_{q, 1}\right)
\end{aligned}
$$

where the first inequality follows from the definition of $\varphi^{*}$ and the second from $1-\alpha=\int \varphi^{*} f_{0} d \lambda_{q, 1} \leq \int \varphi f_{0} d \lambda_{q, 1} . \quad \|$

A second result mirrors Lemma 1 of EMW and bounds the value of $\min _{A} V_{W}(A)$, formalizing the result verbally stated in Section 3.3

Lemma 2. Let $A_{\Lambda, \mathrm{cv}}$ as in Lemma 1 Then for any $A$ that satisfies $\inf _{\theta} C_{\theta}(A) \geq 1-\alpha, V_{W}(A) \geq V_{W}\left(A_{\Lambda, \mathrm{cv}}\right)$.

Proof. The result is immediate from Lemma 1 after noting that $\inf _{\theta} C_{\theta}(A) \geq 1-\alpha \operatorname{implies} \int C_{\theta}(A) d \Lambda(\theta) \geq 1-\alpha$. $\|$

Lemma 2 is useful, as it provides a set of lower bounds (indexed by $\Lambda$ ) on the achievable values of the objective 14. Thus, if a $\Lambda$ can be identified that implies a small lower bound in the sense that a small adjustment to the critical value yields a set with uniform coverage and only marginally larger objective, the problem has been solved as a practical matter. Again following EMW, we denote such a distribution an ALFD.

Definition 2. An $\epsilon$-approximate least favourable distribution $\Lambda^{*}$ is a probability distribution on $\theta$ satisfying

(i) there exists $\mathrm{cv}^{*}$ such that $A_{\Lambda^{*}, \mathrm{cv}^{*}}$ satisfies $\int C_{\theta}\left(A_{\Lambda^{*}, \mathrm{cv}^{*}}\right) d \Lambda^{*}(\theta)=1-\alpha$

(ii) there exists $\mathrm{cv}^{* \epsilon}<\mathrm{cv}^{*}$ such that $\inf _{\theta} \int C_{\theta}\left(A_{\Lambda^{*}, \mathrm{cv}^{*} \epsilon}\right) \geq 1-\alpha$, and $V_{W}\left(A_{\Lambda^{*}, \mathrm{cv}^{*}}\right) \leq V_{W}\left(A_{\Lambda^{*}, \mathrm{cv}^{*}}\right)+\epsilon$.

The strategy is thus to set some small tolerance level $\epsilon$, and to numerically identify an $\epsilon$-ALFD $\Lambda^{*}$. By definition, $A_{\Lambda^{*}, \mathrm{cv} * \epsilon}$ controls coverage uniformly, and invoking Lemma 2 its $W$-weighted average length is at most $\epsilon$ larger than of any prediction set that controls coverage uniformly.

Generalizations of Lemmas 1 and 2 for $A\left(\mathbf{x}^{s}\right)$ additionally restricted to be a superset of some given set $B\left(\mathbf{x}^{s}\right)$ are proven entirely analogously and are omitted for brevity (cf. Müller and Norets, 2012 and Lemma 3 in EMW).

Acknowledgments. We thank Frank Diebold, Graham Elliott, Bruce Hansen, James Stock, Jonathan Wright, three referees, and the Editor, Stéphane Bonhomme, for useful comments and advice. Support was provided by the National Science Foundation through grants SES-0751056 and SES-1226464. Replication files and a supplementary appendix is available at http://www.princeton.edu/ mwatson

\section{Supplementary Data}

Supplementary data are available at Review of Economic Studies online.

\section{REFERENCES}

BALKE, N. S. and GORDON, R. J. (1989), "The Estimation of Prewar Gross National Product: Methodology and New Evidence", Journal of Political Economy, 94, 38-92.

BARRO, R. J. (2006), "Rare Disasters and Asset Markets in the Twentieth Century", The Quarterly Journal of Economics, 121, 823-866.

BERAN, J. (1994), Statistics for Long-Memory Processes (London: Chapman and Hall).

CAMPBELL, J. Y. and VICEIRA, L. M. (1999), "Consumption and Portfolio Decisions When Expected Returns are Time Varying”, Quarterly Journal of Economics, 114, 433-495.

CHIB, S. (1998), "Estimation and Comparison of Multiple Change-Point Models", Journal of Econometrics, 75, $221-241$.

COGLEY, T. and SARGENT, T. (2014), "Measuring Price Level Uncertainty and Stability in the U.S., 1850-2012", Review of Economics and Statistics, forthcoming.

CONGRESSIONAL BUDGET OFFICE (2005), "Quantifying Uncertainty in the Analyis of Long-Term Social Security Projections", (CBO Background paper).

DING, Z., GRANGER, C. W. J. and ENGLE, R. F. (1993), “A Long Memory Property of Stock Market Returns and a New Model", Journal of Empirical Finance, 1, 83-116.

DOORNIK, J. A. and OOMS, M. (2004), "Inference and Forecasting for ARFIMA Models With an Application to U.S. and U.K. Inflation", Studies in Nonlinear Dynamics \& Econometrics, 8, 1-23.

ELLIOTT, G. (2006), "Forecasting with Trending Data", in Elliott, C. W. J. G. G. and Timmerman A. (eds) Handbook of Economic Forecasting, Vol. 1, (Amsterdam: North-Holland), 555-604. 
ELliOTT, G., MÜLLER U. K., and WATSON, M. W. (2015), "Nearly Optimal Tests When a Nuisance Parameter is Present Under the Null Hypothesis", Econometrica, 83, 771-811.

FLECKENSTEIN, M., LONGSTAFF, F. and LUSTIG, H. (2013), "Deflation Risk" (Working Paper No. w19238, NBER).

GRANGER, C. W. and JEON, Y. (2007), "Long-Term Forecasting and Evaluation”, International Journal of Forecasting, 23, 539-551.

HILSHER, J., Raviv A., and Reis, R. (2014), "Inflation Away the Public Debt? An Empirical Assessment" (Working Paper No. w20339, NBER).

KEMP, G. C. R. (1999), “The Behavior of Forecast Errors from a Nearly Integrated AR(1) Model as Both Sample Size and Forecast Horizon Become Large", Econometric Theory, 15, 238-256.

KIEFER, N. and VOGELSANG, T. J. (2005), "A New Asymptotic Theory for Heteroskedasticity-Autocorrelation Robust Tests", Econometric Theory, 21, 1130-1164.

KIEFER, N. M., VOGELSANG T. J., and BUNZEL, H. (2000), "Simple Robust Testing of Regression Hypotheses", Econometrica, 68, 695-714.

KITSUL, Y. and WRIGHT, J. H. (2013), "The Economics of Options-Implied Inflation Probability Density Functions", Journal of Financial Economics, 110, 696-711.

LEE, R. (2011): "The Outlook for Population Growth", Science, 333, 569-573.

LINDGREN, G. (2013), Stationary Stochastic Processes: Theory and Applications (Boca Raton: Chapman \& Hall/CRC). MUIRHEAD, R. J. (1982), Aspects of Multivariate Statistical Theory (Hoboken: Wiley).

MÜLLER, U. K. (2014), "HAC Corrections for Strongly Autocorrelated Time Series", Journal of Business and Economic Statistics, 32, 311-322.

MÜLLER, U. K. and NORETS, A. (2012), "Credibility of Confidence Sets in Nonstandard Econometric Problems", (Working Paper, Princeton University).

MÜLLER, U. K. and WATSON, M. W. (2013), "Testing Models of Low-Frequency Variability”, Econometrica, 76, 979-1016.

MÜLLER, U. K. and WATSON, M. W. (2013), “Low-Frequency Robust Cointegration Testing”, Journal of Econometrics, 174, 66-81.

PASTOR, L. and STAMBAUGH, R. F. (2012), “Are Stocks Really Less Volatile in the Long Run?”, Journal of Finance, LXVII, 431-477.

PESARAN, M. H., PETTENUZZO, D. and TIMMERMANN, A. (2006), "Forecasting Time Series Subject to Multiple Structural Breaks", Review of Economic Studies, 73, 1057-1084.

PESAVENTO, E. and ROSSI, B. (2006), "Small-Sample Confidence Intervals for Multivariate Impulse Response Functions at Long Horizons", Journal of Applied Econometrics, 21, 1135-1155.

PHILLIPS, P. C. B. (1998), "Impulse Response and Forecast Error Variance Asymptotics in Nonstationary VARs", Journal of Econometrics, 83, 21-56.

RAFTERY, A. E., LI, N., SEVCÍKOVA, H., et al. (2012), "Bayesian Probabilistic Population Projections for All Countries", Proceedings of the National Academy of Sciences, USA, 109, 13915-13921.

RIETZ, T. A. (1988), "The Equity Risk Premium a Solution”, Journal of Monetary Economics, 22, 117-131.

ROMER, C. (1989), "The Prewar Business Cycle Reconsidered: New Estimates of Gross National Product, 1869-1908”, Journal of Political Economy, 97, 1-37.

SIEGEL, J. (2007), Stocks for the Long Run: The Definitive Guide to Financial Market Returns and Long Term Investment Strategies (New York: McGraw-Hill).

STOCK, J. H. (1996), "VAR, Error Correction and Pretest Forecasts at Long Horizons", Oxford Bulletin of Economics and Statistics, 58, 685-701.

STOCK, J. H. (1997), "Cointegration, Long-Run Comovements, and Long-Horizon Forecasting", in Kreps D. and Wallis, K. Advances in Econometrics: Proceedings of the Seventh World Congress of the Econometric Society, (Cambridge: Cambridge University Press) 34-60.

STOCK, J. H. and WATSON, M. W. (2002), "Has the Business Cycle Changed and Why?", in Gertler M. and Rogoff K. S. (eds), NBER Macroeconomics Annual 2002, (Cambridge, MA: MIT Press) 159-218.

STOCK, J. H. and WATSON, M. W. (2007), "Why Has Inflation Become Harder to Forecast?", Journal of Money, Credit, and Banking, 39, 3-34.

WALTON, G. and ROCKOFF, H. (2013), History of the American Economy, 12th edn. (Mason: South-Western College Publishing).

WATSON, M. W. (1994), "Business Cycle Durations and Postwar Stabilization of the U.S. Economy”, American Economic Review, 84, 24-46.

WATSON, M. W. (2014), "Inflation Persistence, the NAIRU, and the Great Recession", American Economic Review, 104, 31-36. 